\title{
FINITE EDGE-TRANSITIVE ORIENTED GRAPHS OF VALENCY FOUR WITH CYCLIC NORMAL QUOTIENTS
}

\author{
JEHAN A. AL-BAR, AHMAD N. AL-KENANI, \\ NAJAT MOHAMMAD MUTHANA, AND CHERYL E. PRAEGER
}

\begin{abstract}
We study finite four-valent graphs $\Gamma$ admitting an edge-transitive group $G$ of automorphisms such that $G$ determines and preserves an edgeorientation on $\Gamma$, and such that at least one $G$-normal quotient is a cycle (a quotient modulo the orbits of a normal subgroup of $G$ ). We show on the one hand that the number of distinct cyclic $G$-normal quotients can be unboundedly large. On the other hand existence of independent cyclic $G$-normal quotients (that is, they are not extendable to a common cyclic $G$-normal quotient) places severe restrictions on the graph $\Gamma$ and we classify all examples. We show there are five infinite families of such pairs $(\Gamma, G)$, and in particular that all such graphs have at least one normal quotient which is an unoriented cycle. We compare this new approach with existing treatments for the subclass of weak metacirculant graphs with these properties, finding that only two infinite families of examples occur in common from both analyses. Several open problems are posed.
\end{abstract}

\section{INTRODUCTION}

Finite edge-transitive oriented graphs of valency four have been studied intensively because of their links to maps on Riemann surfaces [6, 8]. They are simple undirected graphs of valency 4 which admit an orientation of their edges determined and preserved by the action of a vertex-transitive and edge-transitive automorphism group. The work of Marušič (summarised in [6, but see also [7, 9, 11]) demonstrated the importance of a certain family of cycles, occurring as subgraphs, for understanding the internal structure of these graphs. This approach has been exploited recently by Marušič and Šparl [10 for the sub-family of weak metacirculants, to give a classification scheme for this sub-family. On the other hand, pursuing a different approach, recent work of the authors in [1] suggests that cycles occurring as normal quotients play a special role. In this paper we study these graphs which have a cycle (oriented or unoriented) as a normal quotient, and classify the graphs with at least two 'independent' cyclic normal quotients (as defined in Definition 1.1). We compare this classification with the analysis in [10] for weak metacirculants in Subsection 1.1

Normal quotients and basic graphs of cycle type: Let $\mathcal{O G}(4)$ denote the family of all pairs $(\Gamma, G)$, where $\Gamma$ is a finite simple connected undirected graph of valency 4 , and $G \leq \operatorname{Aut}(\Gamma)$ is a vertex-transitive and edge-transitive group of

Key words and phrases. edge-transitive graphs, oriented graphs, cyclic quotient graph, transitive group.

This project was funded by the Deanship of Scientific Research (DSR), King Abdulaziz University, Jeddah, under grant no. HiCi/H1433/363-1. The authors, therefore, acknowledge with thanks DSR technical and financial support. 
automorphisms with a specified $G$-orbit $\Delta$ on ordered vertex-pairs consisting of one ordered pair for each edge. In the literature a $G$-action with these properties is said to be $\frac{1}{2}$-transitive. An edge orientation is defined as follows: orient each edge $\{x, y\}$ of $\Gamma$ from $x$ to $y$ if $(x, y) \in \Delta$. Then $\Gamma$ is said to be $G$-oriented. Our notation suppresses the orbit $\Delta$, and we note that the edge-orientation is determined by $G$ up to possibly replacing $\Delta$ by $\{(x, y) \mid(y, x) \in \Delta\}$ (which corresponds to reversing the orientation of each edge).

For $(\Gamma, G) \in \mathcal{O} \mathcal{G}(4)$ with vertex set $X$, and a normal subgroup $N$ of $G$, the normal quotient $\Gamma_{N}$ of $(\Gamma, G)$ has as vertices the $N$-orbits in $X$, and a pair $\{B, C\}$ of distinct $N$-orbits forms an edge of $\Gamma_{N}$ if and only if there is at least one edge $\{x, y\}$ of $\Gamma$ with $x \in B$ and $y \in C$. The quotient $\Gamma_{N}$ is proper if $N \neq 1$ (so that $\Gamma_{N}$ is strictly smaller than $\Gamma$ ). There is a constant $\ell$, independent of the adjacent pair $B, C$, such that each vertex of $B$ is joined by an edge in $\Gamma$ to exactly $\ell$ vertices of $C$, [1. Proposition 3.1]. If $\ell=1$ then the kernel of the $G$-action on $\Gamma_{N}$ is semiregular and hence equal to $N$, and we say that $(\Gamma, G)$ is a normal cover of $\left(\Gamma_{N}, G / N\right)$.

If all edges from $B$ to $C$ have the same $G$-orientation, then $\Gamma_{N}$ inherits a $G$ invariant orientation from $\Gamma$. For any normal subgroup $N$, by [1, Theorem 1.1], either $(\Gamma, G)$ is a normal cover of $\left(\Gamma_{N}, G / N\right)$ and $\left(\Gamma_{N}, G / N\right) \in \mathcal{O G}(4)$, or the quotient $\Gamma_{N}$ is degenerate: consisting of a single vertex (if $N$ is transitive), or a single edge (if the $N$-orbits form the bipartition of a bipartite graph $\Gamma$ ), or $\Gamma_{N}$ is a cycle possibly, but not necessarily, inheriting a $G$-orientation of its edges. Thus, apart from these degeneracies, the family $\mathcal{O G}(4)$ is closed under the normal quotient operation, and much can be learned from studying its 'basic members', namely those pairs $(\Gamma, G) \in \mathcal{O G}(4)$ for which all proper normal quotients are degenerate. A study of these basic pairs was initiated in [1].

In this paper we focus on pairs $(\Gamma, G) \in \mathcal{O G}(4)$ with at least one cyclic normal quotient. The alternative approach in [10] mentioned above also uses certain graph quotients to subdivide the weak metacirculants in $\mathcal{O G}(4)$ into four broad classes. Our discussion in Subsection 1.1 shows that the graph quotients corresponding to two of these four classes can never arise as normal quotients in our sense, and only those for 'Class I' of [10 can be cyclic normal quotients of pairs in $\mathcal{O G}(4)$. Moreover, because of the major focus in [10] on $\frac{1}{2}$-arc-transitive graphs $\Gamma$ (namely those for which $(\Gamma, \operatorname{Aut}(\Gamma)) \in \mathcal{O} \mathcal{G}(4))$, fewer examples are found than in our situation. We make more detailed remarks in Subsection 1.1 (in particular giving definitions of these concepts).

Questions and results: There are many natural questions that arise: how many different cyclic normal quotients can a pair $(\Gamma, G) \in \mathcal{O} \mathcal{G}(4)$ possess? In particular, how many if $(\Gamma, G)$ is basic? Can a (basic) pair $(\Gamma, G)$ have cyclic normal quotients, some of which inherit an edge orientation while others do not, and if so are there restrictions on the numbers of them? We begin by gathering a few observations concerning these questions in Theorem 1. We denote by $C_{r}$ a cycle of length $r$; and we say that a cyclic normal quotient is $G$-oriented or $G$-unoriented if it does, or does not, inherit a $G$-invariant edge orientation, respectively.

Theorem 1. Given a positive integer $n$, there exists

(a) a basic pair $(\Gamma, G) \in \mathcal{O} \mathcal{G}(4)$ with exactly $n$ pairwise non-isomorphic normal quotients, all $G$-oriented cycles;

(b) a basic pair $(\Gamma, G) \in \mathcal{O G}(4)$ with at least $n$ normal quotients, all $G$ oriented cycles of pairwise coprime lengths; 


\begin{tabular}{lllll}
$\bar{\Gamma}$ & $\bar{G}$ & $\bar{\Gamma}_{\bar{M}} ?$ & Conditions on $r, s$ & Reference \\
\hline$\Gamma(r, s)$ & $G(r, s)$ & yes & at least one odd & Con. 2.6 \& Lems. 2.7. 3.3 \\
$\Gamma^{+}(r, s)$ & $G^{+}(r, s)$ & yes & both even & Con. 2.6 \& Lems. 2.7, 3.4 \\
$\Gamma(r, s)$ & $H(r, s)$ & no & $r$ odd, $s$ even & Con. 2.8 \& Lems. 2.9, 3.8 \\
$\Gamma(s, r)$ & $H(s, r)$ & no & $r$ even, $s$ odd & Con. 2.8 \& Lems. 2.9, 3.8 \\
$\Gamma^{+}(r, s)$ & $H^{+}(r, s)$ & no & both even & Con. 2.8 \& Lems. 2.9, 3.7 \\
$\Gamma_{2}(r, s)$ & $G_{2}(r, s)$ & no & both odd & Con. 2.10 \& Lems. 2.11, 3.6 \\
\hline
\end{tabular}

TABle 1. Table for Theorem 2(c)

(c) a pair $(\Gamma, G) \in \mathcal{O G}(4)$ with at least $2 n$ normal quotients $\Gamma_{N_{i}} \cong C_{r_{i}}$ and $\Gamma_{M_{i}} \cong C_{s_{i}}$, for $1 \leq i \leq n$, such that $\operatorname{gcd}\left(r_{i}, r_{j}\right)=\operatorname{gcd}\left(s_{i}, s_{j}\right)=1$ for $i \neq j$, and such that each $\Gamma_{N_{i}}$ is G-oriented while each $\Gamma_{M_{i}}$ is G-unoriented.

The pairs $(\Gamma, G)$ used in the proof of Theorem 1 (c) for $n>1$ are not basic and we do not know of any basic pairs with these properties.

Problem 1. Decide if the number of unoriented cyclic normal quotients of a basic pair $(\Gamma, G) \in \mathcal{O G}(4)$ can be unboundedly large.

The multitude of cyclic normal quotients in the examples examined to prove Theorem 1 are all quotients of one or two particular cyclic normal quotients. For example, in the proof of Theorem 1 (c) using $(\Gamma, G)$ from Construction 2.6, the quotients $\Gamma_{N_{i}}$ are all quotients of a single normal quotient $\Gamma_{N} \cong C_{r}$ with $r$ divisible by all the $r_{i}$, and all the $\Gamma_{M_{j}}$ are quotients of a single normal quotient $\Gamma_{M} \cong C_{s}$ with $s$ divisible by all the $s_{j}$.

Definition 1.1. Two cyclic normal quotients $\Gamma_{M}, \Gamma_{N}$ of $(\Gamma, G) \in \mathcal{O G}(4)$ are independent if the normal quotient $\Gamma_{K}$ is not a cycle, where $K=\tilde{N} \cap \tilde{M}$, with $\tilde{N}, \tilde{M}$ the subgroups consisting of all the elements of $G$ which fix setwise each $N$-orbit, or each $M$-orbit, respectively.

If $\Gamma_{M}, \Gamma_{N}$ are cyclic normal quotients of $(\Gamma, G) \in \mathcal{O G}(4)$ and the normal quotient $\Gamma_{K}$ is a cycle, where $K=\tilde{N} \cap \tilde{M}$, then both $\Gamma_{N}$ and $\Gamma_{M}$ are isomorphic to $(G / K)$ normal quotients of $\Gamma_{K}$, and hence both or neither of them are $G$-oriented, according as $\Gamma_{K}$ is $G$-oriented or not. Thus if one of $\Gamma_{N}, \Gamma_{M}$ is $G$-oriented and the other is $G$-unoriented, then $\Gamma_{N}, \Gamma_{M}$ must be independent. The graphs used to prove Theorem 1(a), (b) do not have independent cyclic normal quotients, but those used to prove Theorem 1(c) do have such quotients. Our main result classifies all pairs $(\Gamma, G) \in \mathcal{O G}(4)$ with independent cyclic normal quotients of given orders.

Theorem 2. Let $(\Gamma, G) \in \mathcal{O G}(4)$, let $x$ be a vertex, and suppose that $(\Gamma, G)$ has independent cyclic normal quotients $\Gamma_{N} \cong C_{r}$ and $\Gamma_{M} \cong C_{s}$, where $r \geq 3, s \geq 3$. Then $G_{x} \cong Z_{2}$, and the following hold:

(a) at least one of $\Gamma_{N}, \Gamma_{M}$ is $G$-unoriented, say $\Gamma_{N}$ is G-unoriented;

(b) $(\Gamma, G) \in \mathcal{O G}(4)$ is a normal cover of $(\bar{\Gamma}, \bar{G}) \in \mathcal{O} \mathcal{G}(4)$, which has independent cyclic normal quotients $\bar{\Gamma}_{\bar{N}} \cong C_{r}$ and $\bar{\Gamma}_{\bar{M}} \cong C_{s}$ such that $\bar{N} \cap \bar{M}=1$;

(c) $\bar{\Gamma}, \bar{G}$ are as in one of the lines of Table 1 , and $\bar{\Gamma}_{\bar{M}}$ (and $\Gamma_{M}$ ) are $G$-oriented if and only if the entry in column 3 is 'yes'.

We give several constructions of families in $\mathcal{O G}(4)$ in Section 2 and prove Theorem 1. Then we prove Theorem 2 in Section 3 . 
Remark (a) Some of the pairs in Theorem 2(c) have larger cyclic normal quotients, for example, if $(\bar{\Gamma}, \bar{G})=(\Gamma(r, s), G(r, s))$ is as in line 1 of Table 1 with $s$ even, then $\bar{\Gamma}_{\bar{N}}=C_{r}$ extends to $\bar{\Gamma}_{\bar{N}_{2}}=C_{2 r}$ (see Lemma 2.7). In this case $\bar{\Gamma}_{\bar{N}_{2}}, \bar{\Gamma}_{\bar{M}}$ are independent for $(\bar{\Gamma}, \bar{G})$, and $\bar{N}_{2} \cap \bar{M}=1$. Applying Theorem 2 to $(\bar{\Gamma}, \bar{G})$ with these cyclic normal quotients of orders $2 r$ and $s$, we find that the pair $(\bar{\Gamma}, \bar{G})$ is given by line 2 of Table 1 Thus $(\Gamma(r, s), G(r, s)) \cong\left(\Gamma^{+}(2 r, s), G^{+}(2 r, s)\right)$ when $r$ is odd and $s$ is even.

(b) For each of the pairs $(\bar{\Gamma}, \bar{G})$ in Theorem $2, \bar{\Gamma}$ is arc-transitive (see Definition 2.1 and Construction 2.10). However there may still be $\frac{1}{2}$-arc-transitive graphs $\Gamma$ with automorphism groups $G$ such that $(\Gamma, G) \in \mathcal{O G}(4)$ has independent cyclic normal quotients $\Gamma_{M}, \Gamma_{N}$, since the normal quotient $\bar{\Gamma}=\Gamma_{M \cap N}$ may admit certain automorphisms making it arc-transitive which do not lift to automorphisms of $\Gamma$. In the next subsection we discuss this possibility further.

Problem 2. Describe the maximal cyclic normal quotients of all the pairs $(\bar{\Gamma}, \bar{G})$ in Table1 and in particular decide whether there are any further relations between these graph families (beyond the isomorphisms given in the remark above.

1.1. Comparison with the approach of Marušič and Šparl [10 for weak metacirculants. The first infinite family of $\frac{1}{2}$-arc transitive graphs, constructed in [3, consisted of 'metacirculant graphs' which had been introduced by Alspach and Parsons [2] in 1982. Several other constructions of $\frac{1}{2}$-arc transitive graphs also turned out to be metacirculants. Recently Marušič and Šparl [10] embarked on a thorough analysis of a (proper) sub-family of $\mathcal{O G}(4)$, called weak metacirculants, with a special focus on those which are $\frac{1}{2}$-arc transitive.

Marušič and Šparl 10] in [10 define a graph $\Gamma$ to be a weak $(m, n)$-metacirculant relative to an ordered pair $(\rho, \lambda)$ of its automorphisms, if $\Gamma$ has $m n$ vertices, the automorphism $\rho$ has $m$ cycles of length $n$ on vertices, the cyclic subgroup $\langle\lambda\rangle$ permutes the $\rho$-cycles transitively, and $\rho^{\lambda}=\rho^{r}$, for some $r$ such that $\operatorname{gcd}(r, n)=1$. The subgroup $\langle\rho, \lambda\rangle$ is thus a metacyclic group which acts transitively on the vertices of $\Gamma$. If, in addition, $\lambda^{m}$ fixes a vertex (in which case $\lambda^{m}$ fixes a vertex in each $\rho$ cycle), then $\Gamma$ is called a metacirculant. A graph may be a weak metacirculant relative to more than one pair $(\rho, \lambda)$ (see Construction 4.2), and according to [10, p. 368], it is an open question whether or not all weak metacirculants are in fact metacirculants (relative perhaps to some other pair of automorphisms).

We classified in Theorem 2 the graph-group pairs $(\Gamma, G) \in \mathcal{O G}(4)$ which have independent cyclic normal quotients. We wish to understand which of them are weak metacirculants relative to pairs of automorphisms lying in $G$. We hope thereby to gain a better understanding of how our normal quotient analysis compares with the analysis in [10] which focuses on alternating cycles. As a bsais for this discussion we make the following assumptions.

Hypothesis 1.1. Let $(\Gamma, G) \in \mathcal{O G}(4)$, and suppose that $\Gamma$ is a weak $(m, n)$-metacirculant relative to $(\rho, \lambda)$, for some $\rho, \lambda \in G$. Let $H:=\langle\rho, \lambda\rangle$ and $R:=\langle\rho\rangle$, so $H \leq G$, the subgroup $R$ is normal in $H$, and $H$ is transitive on the vertices of $\Gamma$.

Since the focus in [10] is on $\frac{1}{2}$-arc transitive graphs, and since no Cayley graph of an abelian group is $\frac{1}{2}$-arc transitive (see [10, beginning of $\left.\S 3\right]$ ), the authors there assume that $H$ is non-abelian. From this they deduce that the number $m$ of $R$-orbits is at least 3 ([10, Proposition 3.2]). We lose little generality if we also assume that 


\begin{tabular}{llllllll}
$\bar{m}$ & $\bar{n}$ & $\bar{\Gamma}$ & $\bar{G}$ & $\bar{\rho}$ & $\bar{\lambda}$ & Conditions on $r, s$ & Name for $\bar{\Gamma}$ in [10] \\
\hline$s$ & $r$ & $\Gamma(r, s)$ & $G(r, s)$ & $\mu$ & $\nu$ & at least one odd & $X_{o}(s, r ; 1)$ \\
$s$ & $r / 2$ & $\Gamma^{+}(r, s)$ & $G^{+}(r, s)$ & $\mu^{2}$ & $\mu \nu$ & both even & $X_{e}(s, r / 2 ; 1,0)$ \\
\hline
\end{tabular}

TABLE 2. Table for Corollary 1.2

$m \geq 3$. The subdivision introduced in [10] of the family of weak metacirculants in $\mathcal{O G}(4)$, and studied further in 4, 13, 14, 15, 16, is defined according to the nature of a certain quotient graph defined modulo the $R$-orbits $X_{i}(1 \leq i \leq m)$ on vertices (that is to say, the $\rho$-cycles). This quotient is slightly different from our graph quotients in that it also encodes, for a vertex $x$ in $X_{i}$, the number of edges from $x$ to vertices in $X_{j}$, for each $j$. Four different kinds of quotients are identified in 10, and these are used to subdivide the weak metacirculants in $\mathcal{O G}(4)$ into four classes, denoted I, II, III, IV. The reason that four different behaviours are observed in 10 is that some of the quotient graphs in [10] do not correspond to a normal quotient of $(\Gamma, G) \in \mathcal{O G}(4)$ for any $G$. In fact, it is only the quotients arising for graphs in Class I of 10, which can possibly occur as cyclic normal quotients in our sense (see Lemma 4.1). Moreover, for $(\Gamma, G) \in \mathcal{O G}(4)$, it is possible to have different choices of $\rho, \lambda \in G$ leading to different quotients $\Gamma_{R}$ of $\Gamma$ which may or may not be normal quotients of $(\Gamma, G)$, and if they are normal quotients, then they may or may not be $G$-oriented. In Construction 4.2 we give explicit examples of metacirculants $(\Gamma, G) \in \mathcal{O G}(4)$ with different pairs $(\rho, \lambda)$ of elements in $G$ illustrating each of these possibilities.

Now we turn to the property of having independent cyclic normal quotients, which is characterised in Theorem 2, In that result we do not assume a priori that the edge-transitive group contains a weak metacirculant subgroup. However, all of the pairs $(\bar{\Gamma}, \bar{G})$ in the outcome of Theorem 2 turn out to be metacirculants. We obtain more Class I weak metacirculants in Theorem 2 than those obtained in [10] because our assumptions are valid for some arc-transitive graphs as well as $\frac{1}{2}$-arc transitive graphs. For example, if $\Gamma$ is $\frac{1}{2}$-arc transitive, then it follows from the proofs of [10, Lemmas 4.2 and 4.3] that the quotient $\Gamma_{R}$ is $G$-oriented, whereas the graphs in lines 3-6 of Table 1 correspond to examples in Theorem 2(c) having a $G$-unoriented normal quotient which can occur as $\Gamma_{R}$ for suitable choices of $\rho, \lambda$.

Our approach can bring additional insights to the work in 10. In the light of our discussion it makes sense to restrict to the Class I weak metacirculants for which $\Gamma_{R}$ is a cyclic $G$-oriented normal quotient of $(\Gamma, G)$. Using Theorem 2 we find all such graphs with independent cyclic normal quotients.

Corollary 1.2. Suppose that Hypothesis 1.1 holds with $\Gamma_{R}$ a cyclic, G-oriented, normal quotient of $(\Gamma, G)$ of length at least 3 . Suppose also that $(\Gamma, G)$ has independent cyclic normal quotients $\Gamma_{N} \cong C_{r}, \Gamma_{M} \cong C_{s}$, as in Theorem 2. Then $(\Gamma, G)$ is a normal cover of $(\bar{\Gamma}, \bar{G}) \in \mathcal{O} \mathcal{G}(4)$ such that $\bar{\Gamma}$ is a weak $(\bar{m}, \bar{n})$-metacirculant relative to $(\bar{\rho}, \bar{\lambda})$ and one of the lines of Table 2 holds.

This corollary is proved in Section 4. The Class I metacirculant graphs which are $\frac{1}{2}$-arc transitive were proved in [10, Theorem 4.1] to be precisely the connected 4valent tightly attached $\frac{1}{2}$-arc transitive graphs (see also [14]). To assist in comparing Corollary 1.2 with that classification we give in Table2 also the names of the graphs $\bar{\Gamma}$ used in [10, Examples 2.1 and 2.2]. 


\section{Constructions And proof the Theorem 1}

In this section we examine several infinite families of graph-group pairs in $\mathcal{O G}(4)$, and describe their cyclic normal quotients. In Subsection 2.6 we prove Theorem 1

2.1. Notation. For fundamental graph theoretic concepts please refer to the book [5]. For a subset $Y$ of the vertex set of a graph $\Gamma$, the induced subgraph $[Y]$ is the graph with vertex set $Y$ and the edges of $[Y]$ are those edges of $\Gamma$ with both vertices in $Y$. An induced subgraph will inherit an edge-orientation from an edge-orientation of $\Gamma$.

A permutation group $N$ on a set $V$ is semiregular if the only element of $N$ fixing a point of $V$ is the identity; also $N$ is regular if it is both transitive and semiregular.

For $(\Gamma, G) \in \mathcal{O G}(4)$, if an edge $\{x, y\}$ is oriented from $x$ to $y$, then we call $y$ an out-neighbour of $x$, and we call $x$ an in-neighbour of $y$. By a neighbour of $x$ we mean an in-neighbour or an out-neighbour.

We say that graph-group pairs $(\Gamma, G)$ and $\left(\Gamma^{\prime}, G^{\prime}\right)$ are isomorphic if there exist a graph isomorphism $f$ from $\Gamma$ to $\Gamma^{\prime}$ and a group isomorphism $\varphi: G \rightarrow G^{\prime}$ such that, for all $x \in V \Gamma$ and $g \in G,\left(x^{g}\right) f=(x f)^{g \varphi}$.

For a group $K$ with an inverse-closed generating set $S$ such that $1_{K} \notin S$ (that is $S^{-1}=\left\{s^{-1} \mid s \in S\right\}$ is equal to $\left.S\right)$, the Cayley graph Cay $(K, S)$ is the graph with vertex set $K$ and edges $\{k, s k\}$ for $k \in K, s \in S$. The facts that $S$ generates $K$ and $S$ is inverse-closed imply that $\operatorname{Cay}(K, S)$ is connected and undirected, respectively. The definition of adjacency implies that $K$ acts faithfully by right multiplication as a vertex-regular group of automorphisms of $\operatorname{Cay}(K, S)$. Also, $S$ is the set of neighbours of the vertex $1_{K}$, and the subgroup of Aut $(K)$ leaving $S$ invariant acts naturally as a subgroup of automorphisms stabilising $1_{K}$.

2.2. Preliminaries on cyclic normal quotients. We first show that oriented and unoriented cyclic normal quotients of $(\Gamma, G) \in \mathcal{O G}(4)$ can be distinguished by the action on vertices of the normal subgroup.

Lemma 2.1. Let $(\Gamma, G) \in \mathcal{O G}(4)$ have a cyclic normal quotient $\Gamma_{N}$, and let $\tilde{N}$ be the subgroup consisting of all elements of $G$ fixing each $N$-orbit setwise.

(a) If $\Gamma_{N}$ is G-unoriented, then $\tilde{N}=N$ and is semiregular on $V \Gamma$.

(b) If $\Gamma_{N}$ is G-oriented, then $\tilde{N}$ contains $G_{x}$, for each vertex $x$.

Proof. Let $x \in V \Gamma$, let $y, y^{\prime}$ be the out-neighbours of $x$, and let $z, z^{\prime}$ be the inneighbours of $x$. Let $B, B^{\prime}$ denote the $N$-orbits containing $x, y$ respectively. Suppose that $\Gamma_{N}=C_{r}$, for some $r \geq 3$.

(a) Suppose that $\Gamma_{N}$ is $G$-unoriented. Then $B^{\prime}$ contains also one of the inneighbours of $x$, say $z$, and the other neighbours $y^{\prime}, z^{\prime}$ lie in a third $N$-orbit distinct from $B, B^{\prime}$. Consider the stabiliser $\tilde{N}_{x}$ of $x$ in $\tilde{N}$. By the definition of $\tilde{N}$, the subgroup $\tilde{N}_{x}$ must fix the $N$-orbit $B^{\prime}$ setwise, and hence must fix the unique outneighbour $y$ of $x$ it contains. Thus $\tilde{N}_{x}$ fixes each of $y$ and $y^{\prime}$. Similarly $\tilde{N}_{x}$ fixes each of $z, z^{\prime}$. It follows from the connectivity of $\Gamma$ that $\tilde{N}_{x}=1$. Thus $\tilde{N}$ is semiregular on $V \Gamma$, and in particular $\tilde{N}=N$.

(b) Now suppose that $\Gamma_{N}$ is $G$-oriented. Then $B^{\prime}$ contains both out-neighbours $y, y^{\prime}$ of $x$. In this case $G$ induces a cyclic group $Z_{r}$ on $\Gamma_{N}$, and the setwise stabiliser $G_{B}$ of $B$ fixes each $N$-orbit setwise, that is to say, $G_{B}=\tilde{N}$. Since $G_{x}<G_{B}$, we have $G_{x}<\tilde{N}$. 
Using this lemma we prove that two oriented cyclic normal quotients cannot be independent.

Lemma 2.2. Let $(\Gamma, G) \in \mathcal{O G}(4)$, and let $\Gamma_{N}=C_{r}, \Gamma_{M}=C_{s}$, for some $r, s \geq 3$, where $N, M$ consist of all elements of $G$ fixing setwise each $N$-orbit, or each $M$ orbit, respectively. If both $\Gamma_{N}$ and $\Gamma_{M}$ are $G$-oriented, then $\Gamma_{N \cap M}=C_{t}$ is $G$ oriented, for some multiple $t$ of $\operatorname{lcm}\{r, s\}$. In particular $\Gamma_{N}, \Gamma_{M}$ are not independent.

Proof. By Lemma 2.1(b), the subgroup $K:=N \cap M$ contains $G_{x}$, for a vertex $x$, and in particular $K \neq 1$. This implies, by [1, Theorem 1.1], that $\Gamma_{K}$ is degenerate, and since it has order at least $\operatorname{lcm}\{r, s\} \geq 3$, it must be a cycle of length a multiple $t$ of $\operatorname{lcm}\{r, s\}$. Since $\Gamma_{N}$ is isomorphic to a quotient of $\Gamma_{K}$, it follows that $\Gamma_{K}$ is $G$-oriented.

2.3. Examples with many cyclic normal quotients. The first family of graphs we consider consists of the lexicographic products $C_{r}\left[2 . K_{1}\right]$ with a natural orientation on their edges.

Construction 2.3. Let $r \geq 3$ and let $\Gamma$ be the graph with vertex set $X=\mathbb{Z}_{r} \times \mathbb{Z}_{2}$ and edges $\left\{(i, j),\left(i+1, j^{\prime}\right)\right\}$ for all $i \in \mathbb{Z}_{r}, j, j^{\prime} \in \mathbb{Z}_{2}$, that is, $\Gamma=C_{r}\left[2 . K_{1}\right]$, the lexicographic product of $C_{r}$ and $2 . K_{1}$. We orient the edges so that $(i, j) \rightarrow\left(i+1, j^{\prime}\right)$, for all $i, j, j^{\prime}$. Let

$$
G=Z_{2} \nmid Z_{r}=\left\{\left(\sigma_{1}, \ldots, \sigma_{r}\right) \tau^{\ell} \mid 0 \leq \ell<r, \text { each } \sigma_{k} \in \mathbb{Z}_{2}\right\}
$$

where $\left(\sigma_{1}, \ldots, \sigma_{r}\right):(i, j) \mapsto\left(i, j+\sigma_{i}\right)$, and $\tau:(i, j) \mapsto(i+1, j)$, and let

$$
B=\left\{\left(\sigma_{1}, \ldots, \sigma_{r}\right) \mid \text { each } \sigma_{k} \in \mathbb{Z}_{2}\right\}=Z_{2}^{r},
$$

the 'base group' of $G$.

By [1, Lemma 3.6], $G$ preserves the edge orientation, $(\Gamma, G) \in \mathcal{O} \mathcal{G}(4)$, and $(\Gamma, G)$ is basic of cycle type. We parametrise all the cyclic normal quotients of $(\Gamma, G)$ with the set of divisors of $r$, and show that $(\Gamma, G)$ does not have independent cyclic normal quotients. A cyclic normal quotient $\Gamma_{N}$ is maximal if there is no normal subgroup $K$ of $G$, contained in $N$, and such that $\Gamma_{K}$ is cyclic of order larger than $\Gamma_{N}$.

Lemma 2.4. Let $r, \Gamma, G, X$ be as in Construction 2.3, and for a divisor $c$ of $r$ let $N(c)=B \cdot\left\langle\tau^{c}\right\rangle$. Then $N(c)$ is normal in $G$ and $\Gamma_{N(c)}=C_{c}$ and is $G$-oriented, if $c \geq 3$, or is $K_{2}$ or $K_{1}$ if $c=2$ or 1 , respectively. Moreover each proper normal quotient of $(\Gamma, G)$ is equal to $\Gamma_{N(c)}$ for some $c$, and $\Gamma_{B}=\Gamma_{N(r)}$ is the unique maximal cyclic normal quotient.

Proof. It was shown in [1, Lemma 3.6] that $\Gamma_{B} \cong C_{r}$ and is $G$-oriented. By definition $N(c)$ contains $B$ and, since $G / B \cong Z_{r}$, the group $N(c)$ is normal in $G$ and $G / N(c) \cong Z_{c}$. Also the graph $\Gamma_{N(c)}$ is isomorphic to the quotient of $\left(\Gamma_{B}, G / B\right)$ relative to the normal subgroup $N(c) / B \cong Z_{r / c}$ of $G / B$, so $\Gamma_{N(c)} \cong C_{c}$ and is $G$ oriented, if $c \geq 3$, and is $K_{2}, K_{1}$ if $c=2,1$ respectively.

Let $N$ be a nontrivial normal subgroup of $G$, and consider $\Gamma_{N}$. It was shown in the proof of [1, Lemma 3.6] that each $N$-orbit on vertices is a union of some $B$-orbits. Thus $B$ fixes each vertex of $\Gamma_{N}$ and so $\Gamma_{N}=\Gamma_{B N}$. Since $G / B \cong Z_{r}$ it follows that $B N=N(c)$ for some divisor $c$ of $r$, and hence $\Gamma_{N}=\Gamma_{N(c)}$. If $c=r$ then $N(r)=B$, and we conclude that $\Gamma_{B}=\Gamma_{N(r)}$ is the unique maximal cyclic normal quotient of $(\Gamma, G)$. 


\begin{tabular}{llll}
$\Gamma$ & $G$ & Generators for $G$ & Conditions on $r, s$ \\
\hline$\Gamma(r, s)$ & $G(r, s)$ & $\mu, \nu, \sigma$ & - \\
$\Gamma(r, s)$ & $H(r, s)$ & $\mu, \sigma \nu, \tau$ & $s$ even \\
$\Gamma^{+}(r, s)$ & $G^{+}(r, s)$ & $\mu^{2}, \mu \nu, \sigma$ & both even \\
$\Gamma^{+}(r, s)$ & $H^{+}(r, s)$ & $\mu^{2}, \sigma \mu \nu, \tau$ & both even \\
\hline
\end{tabular}

TABle 3. Table for Definition 2.1

2.4. Graphs with both independent oriented and unoriented cyclic normal quotients. To prove the second part of Theorem 1, we use a family of graphs given in Definition 2.1. We define an edge-orientation and a group of automorphisms preserving it. We also describe a certain subgraph in some cases. These graphs were considered in [7, Section 3] from the point of view of their alternating cycle structure (see also [9, Example 2.4]).

Recall the concept induced subgraph from Subsection 2.1. Note that, if $k$ is even, then the integers representing a given element of $\mathbb{Z}_{k}$ are either all even, or all odd, and hence in this case elements of $\mathbb{Z}_{k}$ have a well-defined parity. First we define the undirected graphs and subgroups of their automorphism groups.

Definition 2.1. Let $r, s$ be integers, each at least 3. Define the undirected graph $\Gamma(r, s)$ to have vertex set $X:=\mathbb{Z}_{r} \times \mathbb{Z}_{s}$, such that a vertex $(i, j) \in X$ is joined by an edge to each of the four vertices $(i \pm 1, j \pm 1)$. Also, if $r, s$ are both even define

$$
X^{+}:=\{(i, j) \in X \mid i, j \text { of the same parity }\}
$$

and let $\Gamma^{+}(r, s)=\left[X^{+}\right]$, the induced subgraph. Finally, define the following permutations of $X$, for $(i, j) \in X$,

$$
\begin{array}{llll}
\mu:(i, j) & \mapsto(i+1, j), & \nu:(i, j) \mapsto(i, j+1), \\
\sigma:(i, j) \mapsto(-i, j), & \tau:(i, j) \mapsto(-i,-j),
\end{array}
$$

and define the groups as in Table 3, where in lines 3 and 4 ( $r, s$ both even), we identify $\mu, \nu, \sigma, \tau$ with their restrictions to $X^{+}$, and consider the subgroups $G^{+}(r, s)$ and $H^{+}(r, s)$ acting on $X^{+}$.

Also let $\tilde{M}=\langle\mu, \sigma\rangle=D_{2 r}, M=\langle\mu\rangle=Z_{r}, N=\langle\nu\rangle \cong Z_{s}$, and $N^{\prime}=\left\langle\nu^{2}, \tau \sigma \nu\right\rangle$, and note that $G(r, s)=\tilde{M} \times N$ and $H(r, s)=\left(M \times N^{\prime}\right) .\langle\tau\rangle$ (with $s$ even; note that $\left.(\sigma \nu)^{2}=\nu^{2}\right)$.

Let $M_{t}=\left\langle\mu^{t}\right\rangle$ for $t \mid r$, and $N_{t}=\left\langle\nu^{t}\right\rangle$ for $t \mid s$. If $r$ and $s$ are both even, we also consider the following subgroups restricted to their actions on $X^{+}: \tilde{M}^{+}=$ $\left\langle\mu^{2}, \sigma\right\rangle=D_{r}, M^{+}=M_{2}=Z_{r / 2}$, and $N^{+}=N_{2} \cong Z_{s / 2}$.

Recall that, for a graph $\Gamma$, an action of a group $H$ is $\frac{1}{2}$-transitive on $\Gamma$ if $H \leq$ Aut $(\Gamma)$ and $H$ is transitive on the vertices and the edges of $\Gamma$, but is not transitive on arcs.

Lemma 2.5. Let $r, s, \Gamma(r, s), G(r, s), H(r, s), M, N, \Gamma^{+}(r, s), G^{+}(r, s), H^{+}(r, s)$ be as in Definition 2.1. Then

(a) $\Gamma(r, s)$ is connected if at least one of $r, s$ is odd; while if both $r, s$ are even then $\Gamma(r, s)$ has connected components $\left[X^{+}\right]$and $\left[X \backslash X^{+}\right]$, and $\Gamma^{+}(r, s)$ is connected.

(b) $\Gamma(r, s)$ is bipartite if and only if at least one of $r, s$ is even; while if both $r, s$ are even, then $\Gamma^{+}(r, s)$ is bipartite. 
(c) $G(r, s)$, and (if $s$ is even) $H(r, s)$ are $\frac{1}{2}$-transitive on $\Gamma(r, s)$; and if $r, s$ are both even, then $G^{+}(r, s)$ and $H^{+}(r, s)$ are $\frac{1}{2}$-transitive on $\Gamma^{+}(r, s)$.

Proof. The $N$-orbits in $X$ are the sets $B_{i}:=\left\{(i, j) \mid j \in \mathbb{Z}_{s}\right\}$, for $i \in \mathbb{Z}_{r}$. If $s$ is odd, then by the definition of $\Gamma$, for each $i$, each vertex of $B_{i}$ is connected by a path in $\Gamma$ to each vertex of $B_{i+1}$, and hence $\Gamma$ is connected. Suppose then that $s$ is even and define $B_{i}^{\text {even }}=\left\{(i, 2 j) \mid j \in \mathbb{Z}_{s}\right\}$ and $B_{i}^{\text {odd }}=B_{i} \backslash B_{i}^{\text {even }}$, for $i \in \mathbb{Z}_{r}$. Then the definition of $\Gamma$ implies, for each $i$, that each vertex of $B_{i}^{\text {odd }}$ (or $B_{i}^{\text {even }}$ ) is connected by a path in $\Gamma$ to each vertex of $B_{i+1}^{\text {even }}$ (or $B_{i+1}^{\text {odd }}$, respectively). Therefore, if $r$ is odd, $\Gamma$ is connected. On the other hand if $r$ and $s$ are both even, then $\Gamma$ is disconnected with connected components $\left[X^{+}\right]$and $\left[X \backslash X^{+}\right]$. In this case the induced subgraph $\Gamma^{+}$is connected. This proves (a).

If $r$ is even, then it follows from the discussion above that $\cup_{i}$ even $B_{i}$ and $\cup_{i}$ odd $B_{i}$ form the parts of a bipartition of $\Gamma$, and similarly $\Gamma$ is bipartite if $s$ is even (the parts of the bipartition being unions of $M$-orbits). On the other hand if $r, s$ are both odd then $|V \Gamma|$ is odd so $\Gamma$ is not bipartite. If both $r, s$ are even then $\Gamma^{+}$is bipartite with bipartition $\{(i, j) \mid i, j$ even $\},\{(i, j) \mid i, j$ odd $\}$. This proves (b).

It is straightforward to check that each of $\mu, \nu, \sigma, \tau$ preserves the edge set of $\Gamma=\Gamma(r, s)$, so that $G:=G(r, s)$ and $H:=H(r, s)$ lie in Aut $(\Gamma)$. Also, if both $r, s$ are even, then $\mu^{2}, \mu \nu, \sigma, \tau$ all leave $X^{+}$invariant, so $G^{+}:=G^{+}(r, s)$ and $H^{+}:=H^{+}(r, s)$ are contained in $\operatorname{Aut}\left(\Gamma^{+}\right)$, where $\Gamma^{+}=\Gamma^{+}(r, s)$. The subgroup $\langle\mu, \nu\rangle$ of $G$ acts regularly on $X$ and so also, if $s$ is even, does the subgroup $\left\langle\mu, \nu^{2}, \tau \sigma \nu\right\rangle$ of $H$. Hence $G$ and $H$ are vertex-transitive. The stabiliser in $G$, or in $H$, of the vertex $x=(0,0)$ is $\langle\sigma\rangle$, or $\langle\tau\rangle$, respectively. The element $\sigma$ acts on the four neighours of $x$ by interchanging $(1,1)$ and $(-1,1)$ and interchanging $(-1,-1)$ and $(1,-1)$, and the element $\sigma \mu \nu^{-1} \in G$ maps the edge $\{x,(1,1)\}$ to the edge $\{x,(1,-1)\}$. Thus $G$ is edge-transitive but not arc-transitive on $\Gamma$. If $s$ is even, then $\tau$ interchanges $(1,1)$ and $(-1,-1)$ and interchanges $(1,-1)$ and $(-1,1)$, and the element $\tau \mu \sigma \nu \in H$ maps the edge $\{x,(1,1)\}$ to the edge $\{x,(-1,1)\}$. So $H$ also is edge-transitive but not arc-transitive on $\Gamma$. Similar arguments show firstly that (i) the subgroup $\left\langle\mu^{2}, \mu \nu\right\rangle$ of $G^{+}$acts regularly on $X^{+}$, that $G_{x}^{+}=\langle\sigma\rangle$, and that $G^{+}$is $\frac{1}{2}$-transitive on $\Gamma^{+}$; and secondly that (ii) the subgroup $\left\langle\mu^{2}, \sigma \mu \nu\right\rangle$ of $H^{+}$acts regularly on $X^{+}$, that $H_{x}^{+}=\langle\tau\rangle$, and that $H^{+}$is $\frac{1}{2}$-transitive on $\Gamma^{+}$. This proves part (c).

First we define an edge-orientation on the graphs in Definition 2.1 which leads to independent cyclic normal quotients, one oriented and the other not. We note that this family of oriented graphs was studied in [7, p.50-51. Props. 3.2, 3.3] and [8. p.159, Proposition 3.3], where they were characterised by properties of their alternating cycles.

Construction 2.6. Let $r, s, \Gamma(r, s), \Gamma^{+}(r, s)$ be as in Definition 2.1. Define an edgeorientation of $\Gamma(r, s)$ such that $(i, j) \rightarrow(i \pm 1, j+1)$ for each $(i, j) \in X$. If $r, s$ are both even this restricts to an edge-orientation of $\Gamma^{+}(r, s)=\left[X^{+}\right]$.

Lemma 2.7. Let $\Gamma=\Gamma(r, s), G=G(r, s), N, N_{2}, M, \tilde{M}, \Gamma^{+}=\Gamma^{+}(r, s), G^{+}=$ $G^{+}(r, s), N^{+}, M^{+}, \tilde{M}^{+}$be as in Definition 2.1, with edge-orientations of $\Gamma, \Gamma^{+}$as in Construction 2.6.

(a) If at least one of $r, s$ is odd, then $(\Gamma, G) \in \mathcal{O G}(4)$ and $\Gamma_{N}=C_{r}, \Gamma_{\tilde{M}}=\Gamma_{M}=$ $C_{s}$ are independent cyclic normal quotients; $\Gamma_{N}$ is $G$-unoriented while $\Gamma_{M}$ is $G$-oriented. Moreover if $s$ is even then $\Gamma_{N_{2}}=C_{2 r}$ and is $G$-unoriented. 
(b) If both $r, s$ are even, then $\left(\Gamma^{+}, G^{+}\right) \in \mathcal{O G}(4)$ and $\Gamma_{N^{+}}^{+}=C_{r}, \Gamma_{\tilde{M}^{+}}^{+}=\Gamma_{M^{+}}^{+}=$ $C_{s}$ are independent cyclic normal quotients; $\Gamma_{N^{+}}^{+}$is G-unoriented while $\Gamma_{M^{+}}^{+}$is G-oriented.

Proof. By Lemma 2.5 $G, G^{+}$are $\frac{1}{2}$-transitive on $\Gamma, \Gamma^{+}$respectively, and it is straightforward to check that the edge-orientation of Construction 2.6 is preserved in each case. If at least one of $r, s$ is odd, then $\Gamma$ is connected, by Lemma 2.1 and hence $(\Gamma, G) \in \mathcal{O G}(4)$. Similarly if both $r, s$ are even, then $\Gamma^{+}$is connected, by Lemma 2.1 and hence $\left(\Gamma^{+}, G^{+}\right) \in \mathcal{O G}(4)$.

Each of $N, M, \tilde{M}$ is an intransitive normal subgroup of $G$. The $M$-orbits in $X$ are $C_{j}=\left\{(i, j) \mid i \in \mathbb{Z}_{r}\right\}$, for $j \in \mathbb{Z}_{s}$, and are the same as the orbits of $\tilde{M}$, and the $N$-orbits in $X$ are the subsets $B_{i}=\left\{(i, j) \mid j \in \mathbb{Z}_{s}\right\}$, for $i \in \mathbb{Z}_{r}$. Also each of $N^{+}, M^{+}, \tilde{M}^{+}$is a normal subgroup of $G^{+}$and is intransitive on $X^{+}$. The $M^{+}$orbits in $X^{+}$are $C_{j}^{+}:=C_{j} \cap X^{+}$, for $j \in \mathbb{Z}_{s}$, and are the same as the $\tilde{M}^{+}$-orbits in $X^{+}$, and the $N^{+}$-orbits in $X^{+}$are the subsets $B_{i}^{+}:=B_{i} \cap X^{+}$for $i \in \mathbb{Z}_{r}$.

By the definition of $\Gamma$, for each $x=(i, j) \in B_{i}$, each of $B_{i-1}$ and $B_{i+1}$ contains one out-neighbour and one in-neighbour of $x$, and it follows (as in [1, Proposition 3.1(b)]) that $\Gamma_{N}$ is a connected $G$-arc transitive graph of valency 2 and order $r s /\left|B_{i}\right|=r$, so $\Gamma_{N}=C_{r}$ and is $G$-unoriented. Similarly, for each $x=(i, j) \in C_{j}$, both out-neighbours of $x$ lie in $C_{j+1}$ and both in-neighbours of $x$ lie in $C_{j-1}$, so (as in 1, Proposition 3.1(a)]) $\Gamma_{M}=\Gamma_{\tilde{M}}$ is a connected, $G$-oriented, $G$-edge transitive graph of valency 2 and order $r s /\left|C_{j}\right|=s$, that is, $\Gamma_{M}=C_{s}$ and is $G$-oriented. If in addition $s$ is even (so $r$ is odd) then each $N$-orbit $B_{i}$ is the union of two $N_{2}$-orbits, namely $B_{i}^{\text {even }}=\{(i, j) \mid j$ even $\}$ and $B_{i}^{\text {odd }}=\{(i, j) \mid j$ odd $\}$. For $j$ even, the vertex $x=(i, j)$ has one out-neighbour in each of $B_{i-1}^{\text {odd }}$ and $B_{i+1}^{\text {odd }}$, and for $j$ odd, $(i, j)$ has one out-neighbour in each of $B_{i-1}^{\text {even }}$ and $B_{i+1}^{\text {even }}$. Since $r$ is odd, it follows that $\Gamma_{N_{2}}$ is the cycle $C_{2 r}$ and is $G$-unoriented. Part (a) now follows from Definition 1.1 since $N \cap \tilde{M}=1$. Part (b) also follows since the $B_{i}^{+}=B_{i} \cap X^{+}, C_{j}^{+}=C_{j} \cap X^{+}$, $\tilde{M}^{+}=\tilde{M} \cap G^{+}, M^{+}=M \cap G^{+}, N^{+}=N \cap G^{+}$and $\tilde{M}^{+} \cap N^{+}=1$.

2.5. Graphs with independent unoriented cyclic normal quotients. We now give two constructions of oriented graphs with independent unoriented cyclic normal quotients. The graphs in the first construction are the graphs $\Gamma(r, s)$ and $\Gamma^{+}(r, s)$ of Definition 2.1 with a different edge-orientation from that in Construction 2.6.

Construction 2.8. Let $r, s$ be positive integers, with $r \geq 3$, and $s$ even, $s \geq 4$, and recall the graph $\Gamma(r, s)$ of Definition 2.1 with vertex set $X=\mathbb{Z}_{r} \times \mathbb{Z}_{s}$. Define an edge-orientation as follows.

$$
\begin{aligned}
& \text { if } j \text { is even then } \quad(i, j) \rightarrow(i+1, j+1) \text { and } \quad(i, j) \rightarrow(i-1, j-1) \text {, } \\
& \text { if } j \text { is odd then } \quad(i, j) \rightarrow(i+1, j-1) \text { and } \quad(i, j) \rightarrow(i-1, j+1) \text {. }
\end{aligned}
$$

In other words, $(i, j) \rightarrow\left(i+1, j+(-1)^{j}\right)$ and $(i, j) \rightarrow\left(i-1, j-(-1)^{j}\right)$. Note that this orientation is well-defined since elements of $\mathbb{Z}_{s}$ have a well-defined parity as $s$ is even. If $r$ and $s$ are both even, then this edge-orientation restricts to an edge-orientation of $\Gamma^{+}(r, s)=\left[X^{+}\right]$.

Lemma 2.9. Let $s$ be even, $s \geq 4$, and let $r \geq 3$. Let $\Gamma=\Gamma(r, s), H=H(r, s)$, $N^{\prime}, M$, and, if $r$ is even, also $\Gamma^{+}=\Gamma^{+}(r, s), H^{+}=H^{+}(r, s), N^{+}, M^{+}$, be as in Definition 2.1, with edge-orientations of $\Gamma, \Gamma^{+}$as in Construction 2.8. 
(a) If $r$ is odd, then $H$ preserves the edge-orientation of $\Gamma,(\Gamma, H) \in \mathcal{O G}(4)$, and $\Gamma_{N^{\prime}}=C_{r}, \Gamma_{M} \cong C_{s}$ are independent, $H$-unoriented, cyclic normal quotients.

(b) If $r$ is even, then $H^{+}$preserves the edge-orientation of $\Gamma^{+},\left(\Gamma^{+}, H^{+}\right) \in$ $\mathcal{O G}(4)$, and $\Gamma_{N^{+}}^{+}=C_{r}, \Gamma_{M^{+}}^{+} \cong C_{s}$ are independent, $H^{+}$-unoriented, cyclic normal quotients.

Proof. It is easy to check that each of $\mu, \nu^{2}, \tau$ preserves the edge-orientation of $\Gamma$, and with a little care, that $\sigma \nu$ does also. Thus $H$ preserves the edge-orientation of $\Gamma$, and $H^{+}$preserves the edge-orientation of $\Gamma^{+}$.

(a) Suppose that $r$ is odd. Then by Lemma 2.5 $\Gamma$ is connected and $H$ acts $\frac{1}{2}$-transitively, so $(\Gamma, H) \in \mathcal{O G}(4)$. Now $M, N^{\prime}$ are normal subgroups of $H$. The $N^{\prime}$-orbits are $B_{i}=\left\{(i, j) \mid j \in \mathbb{Z}_{s}\right\}$, for $i \in \mathbb{Z}_{r}$. Let $x^{\prime}=(i, j) \in B_{i}$. Then each of $B_{i-1}$ and $B_{i+1}$ contains one out-neighbour and one in-neighbour of $x^{\prime}$. Hence, as in [1, Proposition 3.1(b)], $\Gamma_{N^{\prime}}$ is a connected $H$-arc transitive graph of valency 2 and order $r s /\left|B_{i}\right|=r$, so $\Gamma_{N^{\prime}} \cong C_{r}$ and is $H$-unoriented. Similarly the $M$-orbits are $C_{j}=\left\{(i, j) \mid i \in \mathbb{Z}_{r}\right\}$, for $j \in \mathbb{Z}_{s}$, and for each $x^{\prime}=(i, j) \in C_{j}$, each of $C_{j-1}$ and $C_{j+1}$ contains one out-neighbour and one in-neighbour of $x^{\prime}$, and so $\Gamma_{M} \cong C_{s}$ and is $H$-unoriented. By Lemma 2.1 $N^{\prime}, M$ are the kernels of the $H$-actions on $\Gamma_{N^{\prime}}, \Gamma_{M}$, respectively. Since $N^{\prime} \cap M=1$, the quotients $\Gamma_{N^{\prime}}, \Gamma_{M}$ are independent. This proves part (a).

(b) Suppose now that $r$ is even, $r \geq 4$. Then by Lemma 2.1, $\Gamma^{+}$is connected and $H^{+}$acts $\frac{1}{2}$-transitively, so $\left(\Gamma^{+}, H^{+}\right) \in \mathcal{O} \mathcal{G}(4)$. Now $M^{+}, N^{+}$are normal subgroups of $H^{+}$. The $N^{+}$-orbits in $X^{+}$(with $X^{+}$as in Definition 2.1) are $B_{i} \cap X^{+}$, for $i \in \mathbb{Z}_{r}$. Let $x^{\prime}=(i, j) \in B_{i} \cap X^{+}$. Then each of $B_{i-1} \cap X^{+}$and $B_{i+1} \cap X^{+}$contains one out-neighbour and one in-neighbour of $x^{\prime}$. Hence, as in [1, Proposition 3.1(b)], $\Gamma_{N^{+}}^{+}$ is a connected $H^{+}$-arc transitive graph of valency 2 and order $\left|X^{+}\right| /\left|B_{i} \cap X^{+}\right|=r$, so $\Gamma_{N^{+}}^{+} \cong C_{r}$ and is $H^{+}$-unoriented. Similarly the $M^{+}$-orbits are $C_{j} \cap X^{+}$, for $j \in \mathbb{Z}_{s}$, and for each $x^{\prime}=(i, j) \in C_{j} \cap X^{+}$, each of $C_{j-1} \cap X^{+}$and $C_{j+1} \cap X^{+}$ contains one out-neighbour and one in-neighbour of $x^{\prime}$, and so $\Gamma_{M^{+}}^{+} \cong C_{s}$ and is $H^{+}$-unoriented. By Lemma 2.1, $\mathrm{N}^{+}, \mathrm{M}^{+}$are the kernels of the $\mathrm{H}^{+}$-actions on $\Gamma_{N^{+}}^{+}, \Gamma_{M^{+}}^{+}$, respectively. Since $N^{+} \cap M^{+}=1$, the quotients $\Gamma_{N^{+}}^{+}, \Gamma_{M^{+}}^{+}$are independent.

The graphs in the final construction are standard double covers of the graphs $\Gamma(r, s)$ of Definition 2.1.

Definition 2.2. The standard double cover of a graph $\Gamma$ with vertex set $X$ is the graph $\Gamma_{2}$ with vertex set $X_{2}=\left\{x_{\delta} \mid x \in X, \delta \in \mathbb{Z}_{2}\right\}$ such that $\left\{x_{\delta}, y_{\delta^{\prime}}\right\}$ is an edge if and only if $\delta \neq \delta^{\prime}$ and $\{x, y\}$ is an edge of $\Gamma$.

Note that $\Gamma_{2}$ has the same valency as $\Gamma$ and twice the number of vertices.

Construction 2.10. Let $r, s$ be positive integers, with $r, s \geq 3$, and let $\Gamma_{2}(r, s)$ be the standard double cover of the graph $\Gamma(r, s)$ of Definition 2.1. so $\Gamma_{2}(r, s)$ has vertex set $X_{2}$ as in Definition 2.2. Define an orientation on the edges of $\Gamma_{2}(r, s)$ as follows.

$$
\begin{array}{rlrlrl}
(i, j)_{0} & \rightarrow(i+1, j+1)_{1} & \text { and } & & (i, j)_{0} & \rightarrow(i-1, j-1)_{1} \\
(i, j)_{1} & \rightarrow(i+1, j-1)_{0} & \text { and } & (i, j)_{1} & \rightarrow(i-1, j+1)_{0} .
\end{array}
$$


In other words, $(i, j)_{\delta} \rightarrow\left(i+1, j+(-1)^{\delta}\right)_{\delta+1}$ and $(i, j)_{\delta} \rightarrow\left(i-1, j-(-1)^{\delta}\right)_{\delta+1}$. We extend the automorphisms defined in Definition 2.1 to maps on $X_{2}$ as follows. For $(i, j)_{\delta} \in X_{2}$,

$$
\begin{array}{rlrl}
\mu:(i, j)_{\delta} & \mapsto(i+1, j)_{\delta}, & \nu:(i, j)_{\delta} \mapsto(i, j+1)_{\delta}, \\
\sigma:(i, j)_{\delta} \mapsto(i,-j)_{\delta+1}, & \tau:(i, j)_{\delta} \mapsto(-i,-j)_{\delta}
\end{array}
$$

and let $G_{2}(r, s)=\langle\mu, \nu, \sigma, \tau\rangle=M \times N$, where $M=\langle\mu, \sigma \tau\rangle \cong D_{2 r}$, and $N=$ $\langle\nu, \sigma\rangle \cong D_{2 s}$.

Lemma 2.11. Let $r, s, \Gamma=\Gamma_{2}(r, s), G=G_{2}(r, s)$ be as in Construction 2.10. Then $G$ preserves the edge-orientation, $\Gamma$ is connected if and only if $r, s$ are both odd, and in this case $(\Gamma, G) \in \mathcal{O G}(4)$, and $\Gamma_{N}=C_{r}, \Gamma_{M}=C_{s}$ are independent cyclic normal quotients, and each is $G$-unoriented.

Proof. Careful but straightforward checking shows that each of the generators of $G$ preserves edges, and that each preserves the edge orientation of $\Gamma$. It is well known and easily proved that a standard double cover of a graph $\Sigma$ is connected if and only if $\Sigma$ is connected and not bipartite. It follows therefore from Lemma 2.5 that $\Gamma$ is connected if and only if $r, s$ are both odd. Suppose this is the case.

The subgroup $\langle\mu, \nu, \sigma\rangle$ is normal in $G$ of index 2 , and is regular on vertices. The stabiliser $G_{x}$ of the vertex $x=(0,0)_{0}$ is $\langle\tau\rangle$, which interchanges the out-neighbours $(1,1)_{1}$ and $(-1,-1)_{1}$, and the in-neighbours $(1,-1)_{1}$ and $(1,-1)_{1}$ of $x$. Thus $G$ is $\frac{1}{2}$-transitive on $\Gamma$ and preserves the edge-orientation so $(\Gamma, G) \in \mathcal{O G}(4)$.

The $N$-orbits in $X_{2}$ are the subsets $B_{i}=\left\{(i, j)_{\delta} \mid j \in \mathbb{Z}_{s}, \delta \in \mathbb{Z}_{2}\right\}$, for $i \in \mathbb{Z}_{r}$. Each vertex in $B_{i}$ has one out-neighbour and one in-neighbour in $B_{i+\varepsilon}$, for $\varepsilon= \pm 1$. It follows that $\Gamma_{N}=C_{r}$ and is $G$-unoriented. Similarly the $M$-orbits in $X_{2}$ are the subsets $C_{j}=\left\{(i, j)_{\delta} \mid i \in \mathbb{Z}_{r}, \delta \in \mathbb{Z}_{2}\right\}$, for $j \in \mathbb{Z}_{s}$. Each vertex in $C_{j}$ has one out-neighbour and one in-neighbour in $C_{j+\varepsilon}$, for $\varepsilon= \pm 1$, and hence $\Gamma_{M}=C_{s}$ and is $G$-unoriented. By Lemma 2.1, $N, M$ are the kernels of the actions of $G$ on $\Gamma_{N}, \Gamma_{M}$ respectively, and since $M \cap N=1$ it follows that $\Gamma_{N}, \Gamma_{M}$ are independent.

2.6. Proof of Theorem 1, Let $n$ be a positive integer.

(a) Let $r, \Gamma, G$ be as in Construction 2.3. Choose an odd prime $p$ and set $r=$ $p^{n}$. By [1, Lemma 3.6], $(\Gamma, G)$ is basic in $\mathcal{O G}(4)$, and by Lemma 2.4 its normal quotients relative to intransitive, nontrivial normal subgroups of $G$ are precisely the $G$-oriented quotients $\Gamma_{N(c)}=C_{c}$, for $c \mid r$ and $c>1$. There are precisely $n$ possibilities, namely for $c=p^{i}$ with $i=1, \ldots, n$.

(b) Again let $r, \Gamma, G$ be as in Construction 2.3, and this time choose $n$ distinct odd primes $p_{1}<p_{2}<\cdots<p_{n}$ and take $r=\prod_{i} p_{i}$. Again $(\Gamma, G)$ is basic in $\mathcal{O G}(4)$ of cycle type, by [1, Lemma 3.6], and by Lemma 2.4, we have $G$-oriented normal quotients $\Gamma_{N\left(p_{i}\right)}=C_{p_{i}}$ relative to $N\left(p_{i}\right)$, for $i=1, \ldots, n$.

(c) Choose $2 n$ odd primes $p_{1}<p_{2}<\cdots<p_{n}$ and $q_{1}<q_{2}<\cdots<q_{n}$ (where some $p_{i}, q_{j}$ may be equal), and take $r=\prod_{i} p_{i}$ and $s=\prod_{i} q_{i}$ in Construction 2.6. By Lemma 2.7 $(\Gamma, G) \in \mathcal{O G}(4)$, and $(\Gamma, G)$ has a cyclic $G$-oriented normal quotient $\Gamma_{N}=C_{r}$, and a cyclic $G$-unoriented normal quotient $\Gamma_{M}=C_{s}$. For $i=1, \ldots, n$, consider $N_{p_{i}}=\left\langle\nu^{p_{i}}\right\rangle$ and $M_{q_{i}}=\left\langle\mu^{q_{i}}\right\rangle$, as in Definition 2.1. and note that $N_{p_{i}} \times M$ and $N \times M_{q_{i}}$ are both normal in $G$ and intransitive on vertices. The normal quotients $\Gamma_{N_{p_{i}} \times M}$ and $\Gamma_{N \times M_{q_{i}}}$ are isomorphic to quotients of $\Gamma_{M}$ and $\Gamma_{N}$, and are 
in fact isomorphic to $C_{p_{i}}$ and $C_{q_{i}}$, respectively, with the former $G$-oriented and the latter $G$-unoriented. This completes the proof.

\section{Proof of Theorem 2}

In this section we analyse the structure of pairs $(\Gamma, G) \in \mathcal{O G}(4)$ with independent cyclic normal quotients. The first lemma yields a proof of parts (a) and (b) of Theorem 2

Lemma 3.1. Let $(\Gamma, G) \in \mathcal{O G}(4)$, and suppose that $\Gamma_{N}, \Gamma_{M}$ are independent cyclic normal quotients, where $N, M$ consists of all elements of $G$ fixing setwise each $N$-orbit, or each $M$-orbit, respectively. Let $\bar{\Gamma}=\Gamma_{N \cap M}, \bar{G}=G /(N \cap M), \bar{N}=$ $N /(N \cap M)$ and $\bar{M}=M /(N \cap M)$.

(a) Then one of $\Gamma_{N}, \Gamma_{M}$ is $G$-unoriented, say $\Gamma_{N}=C_{r}$ for some $r \geq 3$, and the other $\Gamma_{M}=C_{s}$, for some $s \geq 3$, may be $G$-oriented or $G$-unoriented.

(b) The quotient $(\bar{\Gamma}, \bar{G}) \in \mathcal{O} \mathcal{G}(4)$ has independent cyclic normal quotients $\bar{\Gamma}_{\bar{N}} \cong$ $C_{r}$ and $\bar{\Gamma}_{\bar{M}} \cong \Gamma_{M}$ such that $\bar{N} \cap \bar{M}=1$, and $(\Gamma, G)$ is a normal cover of $(\bar{\Gamma}, \bar{G})$.

(c) If $N \cap M=1$, then the map $\varphi: g \mapsto\left(g^{\Gamma_{N}}, g^{\Gamma_{M}}\right)$ defines a group monomorphism from $G$ to $D_{2 r} \times A$, such that $G \varphi \pi_{1}=D_{2 r}, G \varphi \pi_{2}=A$, where $\pi_{1}, \pi_{2}$ are the natural projection maps of $D_{2 r} \times A$ on $D_{2 r}, A$ respectively, and $A=D_{2 s}$ or $Z_{s}$ according as $\Gamma_{M}$ is $G$-unoriented or $G$-oriented, respectively.

Proof. Part (a) follows from Lemma 2.2. By Definition 1.1, $\bar{\Gamma}=\Gamma_{N \cap M}$ is not a cycle, and hence, by [1, Theorem 1.1], $(\Gamma, G)$ is a normal cover of $(\bar{\Gamma}, \bar{G})$ and $(\bar{\Gamma}, \bar{G}) \in \mathcal{O G}(4)$. Now $\bar{N}$ and $\bar{M}$ are normal subgroups of $\bar{G}$, and by construction the corresponding $\bar{G}$-normal quotients satisfy $\bar{\Gamma}_{\bar{N}} \cong \Gamma_{N}$ and $\bar{\Gamma}_{\bar{M}} \cong \Gamma_{M}$. These quotients are independent since $\bar{N} \cap \bar{M}=1$. Thus part (b) is proved.

If $N \cap M=1$ then the map $\varphi$ is a monomorphism from $G$ to $\operatorname{Aut}\left(\Gamma_{N}\right) \times$ $\operatorname{Aut}\left(\Gamma_{M}\right)=D_{2 r} \times A$, and $G \varphi \pi_{1}=G^{\Gamma_{N}}=D_{2 r}, G \varphi \pi_{2}=G^{\Gamma_{M}}=A$.

Parts (a) and (b) of Theorem 2 follow from Lemma 3.1. Also, from part (b), $(\Gamma, G)$ is a normal cover of $(\bar{\Gamma}, \bar{G})$, and so the order of the vertex stabiliser $G_{x}$ is equal to the order of a stabiliser in $\bar{G}$ of a vertex of $\bar{\Gamma}$. Thus (even to prove that stabilisers have order 2) it is sufficient to consider the case where $N \cap M=1$. We therefore make this assumption from now on. We use the following notation.

Notation 3.1. We assume that $(\Gamma, G) \in \mathcal{O G}(4)$, and that $\Gamma_{N}=C_{r}(G$-unoriented), $\Gamma_{M}=C_{s}$, and $A=\operatorname{Aut}\left(\Gamma_{M}\right), \varphi, \pi_{1}, \pi_{2}$ are as in Lemma 3.1, with $N \cap M=1$. We use the notation introduced in the proof of Lemma 2.1. so $x \in V \Gamma$ has outneighbours $y, y^{\prime}$ and in-neighbours $z, z^{\prime}$. We let $B=x^{N}, B^{+}=y^{N}, B^{-}=\left(y^{\prime}\right)^{N}$, the pairwise distinct $N$-orbits containing $x, y, y^{\prime}$, and $C=x^{M}, C^{+}=y^{M}, C^{-}=\left(y^{\prime}\right)^{M}$, the $M$-orbits containing $x, y, y^{\prime}$ (so $C^{+}=C^{-}$if $\Gamma_{M}$ is $G$-oriented). Write

$$
\operatorname{Aut}\left(\Gamma_{N}\right)=\left\langle a, c \mid a^{r}=c^{2}=1, a^{c}=a^{-1}\right\rangle \cong D_{2 r}
$$

where $a$ maps $B$ to $B^{+}$and $c$ fixes $B$. We also write

$$
\operatorname{Aut}\left(\Gamma_{M}\right)=A=\left\langle b, d \mid b^{s}=d^{2}=1, b^{d}=b^{-1}\right\rangle \cong D_{2 s} \text { or }\left\langle b \mid b^{s}=1\right\rangle \cong Z_{s}
$$

according as $\Gamma_{M}$ is $G$-unoriented or $G$-oriented, respectively, and $b$ maps $C$ to $C^{+}$ and (if $\Gamma_{M}$ is $G$-unoriented) $d$ fixes $C$. 
3.1. Case $\Gamma_{M}$ is $G$-oriented. First we derive information about generators of $G \varphi$.

Lemma 3.2. Using Notation 3.1, and assuming that $\Gamma_{M}$ is G-oriented,

(a) $G_{x}=\langle h\rangle \cong Z_{2}$ is such that $h \varphi=(c, 1)$.

(b) The following all hold, where either $t=1$, or $t=2$ divides $\operatorname{gcd}(r, s)$ :

$$
|G|=\frac{2 r s}{t},|V \Gamma|=\frac{r s}{t}, N \varphi=\left\langle\left(1, b^{t}\right)\right\rangle, M \varphi=\left\langle\left(a^{t}, 1\right),(c, 1)\right\rangle,
$$

and $G \varphi=\left\langle\left(a^{i}, b\right), M \varphi\right\rangle$, where either $i=0$ or $i=t-1=1$.

Proof. Since $G$ is edge-transitive on $\Gamma, G_{x} \neq 1$. Let $h \in G_{x} \backslash\{1\}$. Then $h$ fixes both $B$ and $C$ setwise and so $h^{\Gamma_{N}} \in\langle c\rangle$ and $h^{\Gamma_{M}}=1$. Since $h \neq 1$ it follows that $h \varphi=(c, 1)$ and $G_{x}=\langle h\rangle \cong Z_{2}$, proving part (a).

Note that $M$ contains $h$, by Lemma 2.1. Since $M \varphi \leq\langle a, c\rangle \times 1$, it follows that $M \varphi=\left\langle\left(a^{t}, 1\right),(c, 1)\right\rangle$, for some $t \mid r$. Hence $|M|=\frac{2 r}{t},|G|=\frac{2 r s}{t}$, and $|V \Gamma|=\frac{r s}{t}$. Also $N \varphi \leq 1 \times\langle b\rangle$ so $N \varphi=\left\langle\left(1, b^{\ell}\right)\right\rangle$ for some $\ell \mid s$, and we have $|N|=\frac{s}{\ell},|G|=\frac{2 r s}{\ell}$. Therefore $\ell=t$ divides $\operatorname{gcd}(r, s)$. Since $G \varphi \pi_{2}=\langle b\rangle, G$ contains an element $g$ such that $g \varphi \pi_{2}=b$. All such elements satisfy $g \varphi=\left(a^{i} c^{\delta}, b\right)$ for some $i, \delta$. We may replace $g$ by $g m$ for some $m \in M$, and assume that $\delta=0$ and that $0 \leq i<t$. It remains to prove that $t \leq 2$. To see this, note that $G \varphi$ contains $\left(a^{i}, b\right) \cdot\left(a^{i}, b\right)^{(c, 1)}=$ $\left(a^{i}, b\right) \cdot\left(a^{-i}, b\right)=\left(1, b^{2}\right)$, which lies in $N \varphi$.

We consider the cases $t=1$ and $t=2$ separately. Recall the concepts of regular, Cayley graph, and isomorphism of graph-group pairs from Subsection 2.1

Lemma 3.3. Under the assumptions of Lemma 3.2, if $t=1$ then at least one of $r, s$ is odd and $(\Gamma, G)$ is isomorphic to the graph-group pair $(\Gamma(r, s), G(r, s))$ in Construction 2.6.

Proof. Suppose that $t=1$ and identify $G$ with $G \varphi$. Then $|V \Gamma|=r s$ and $G=\langle a, c\rangle \times$ $\langle b\rangle$. Moreover the group $K=\langle(a, 1)\rangle \times\langle(1, b)\rangle$, is normal in $G$, acts regularly on $V \Gamma$, and $G$ is the semidirect product $K . G_{x}$. By [1, Remark 4.1 and Lemma 4.2], we may assume that $\Gamma=\operatorname{Cay}\left(K, S_{0} \cup S_{0}^{-1}\right)$ for a 2-element generating set $S_{0}$ for $K$ such that $S_{0} \cap S_{0}^{-1}=\emptyset$, and we may identify $x=1_{K}, S_{0}=\left\{y, y^{\prime}\right\}$, and $S_{0}^{-1}=\left\{z, z^{\prime}\right\}$. The group $K$ acts by right multiplication and $G_{x}=\langle(c, 1)\rangle \leq \operatorname{Aut}(K)$ acts naturally on $V \Gamma=K$. Now $y=\left(a^{i}, b^{j}\right)$ for some $i \in \mathbb{Z}_{r}, j \in \mathbb{Z}_{s}$, and so $y^{\prime}=y^{(c, 1)}=\left(a^{-i}, b^{j}\right)$. In particular $i \neq 0$ since $y^{\prime} \neq y$.

Since $N \leq K$, the $N$-orbits are the cosets $N\left(a^{k}, 1\right)$ for $k \in \mathbb{Z}_{r}$, and as in Notation 3.1. $(a, 1)$ maps $B=1^{N}=N$ to $B^{+}=y^{N}=N\left(a^{i}, 1\right)$. However $(a, 1)$ maps $B$ to $N(a, 1)$ and hence $i=1$. Also $M \cap K=\langle(a, 1)\rangle$ is transitive on each $M$-orbit, and the $M$-orbits are the cosets $M\left(1, b^{k}\right)$ for $k \in \mathbb{Z}_{s}$. As in Notation 3.1, $(1, b)$ maps $C=1^{M}=M$ to $C^{+}=y^{M}=M\left(1, b^{j}\right)$. However $(1, b)$ maps $C$ to $M(1, b)$ and hence $j=1$. Thus $S_{0}=\left\{(a, b),\left(a^{-1}, b\right)\right\}$, and since $K=\left\langle S_{0}\right\rangle$, at least one of $r, s$ must be odd.

It follows that each vertex $\left(a^{k}, b^{\ell}\right)$ has out-neighbours $\left(a^{k \pm 1}, b^{\ell+1}\right)$, and so the map $f:\left(a^{k}, b^{\ell}\right) \mapsto(k, \ell)$ defines a graph isomorphism from $\Gamma$ to the graph $\Gamma(r, s)$ of Construction 2.6. Also the map $(a, 1) \mapsto \mu,(1, b) \mapsto \nu,(c, 1) \mapsto \sigma$ extends to an isomorphism $\varphi^{\prime}$ from $G$ to the group $G(r, s)$ of Construction 2.6. and $f, \varphi^{\prime}$ define an isomorphism from $(\Gamma, G)$ to $(\Gamma(r, s), G(r, s))$. 
Lemma 3.4. Under the assumptions of Lemma 3.2, if $t=2$ then both $r$ and $s$ are even, and $(\Gamma, G)$ is isomorphic to the graph-group pair $\left(\Gamma^{+}(r, s), G^{+}(r, s)\right)$ in Construction 2.6.

Proof. Suppose that $t=2$ and identify $G$ with $G \varphi$. Then $r, s$ are both even, $|V \Gamma|=\frac{r s}{2}$, and $G=\left\langle\left(a^{i}, b\right),\left(a^{2}, 1\right),(c, 1)\right\rangle$ of order $r s$, where $i=0$ or 1 , by Lemma 3.2. Moreover since $G \pi_{1}=\langle a, c\rangle$ it follows that $i=1$. Then the group $K:=\left\langle\left(a^{2}, 1\right),(a, b)\right\rangle$ is a subgroup of $G$ of index 2 , and as $G_{x}=\langle(c, 1)\rangle \cong Z_{2}$, we have $K_{x}=1$ and $K$ acts regularly on $V \Gamma$. As in Lemma 3.3, we may assume that $\Gamma=\operatorname{Cay}\left(K, S_{0} \cup S_{0}^{-1}\right)$ for a 2 -element generating set $S_{0}$ for $K$ such that $S_{0} \cap S_{0}^{-1}=\emptyset$, and we may identify $x=1_{K}, S_{0}=\left\{y, y^{\prime}\right\}$, and $S_{0}^{-1}=\left\{z, z^{\prime}\right\}$. The group $K$ acts by right multiplication and $G_{x}=\langle(c, 1)\rangle \leq \operatorname{Aut}(K)$ acts naturally on $V \Gamma$. Thus $y=\left(a^{j}, b^{k}\right)$ for some $j \in \mathbb{Z}_{r}, k \in \mathbb{Z}_{s}$ of the same parity, and $y^{\prime}=y^{(c, 1)}=\left(a^{-j}, b^{k}\right)$.

Since $N \leq K$, the $N$-orbits are the $\operatorname{cosets} N\left(a^{\ell}, 1\right)$ for even $\ell \in \mathbb{Z}_{r}$, and $N\left(a^{\ell}, b\right)$ for odd $\ell \in \mathbb{Z}_{r}$. As in Notation 3.1. $(a, b) \in K$ and maps $B=1^{N}=N$ to $B^{+}=y^{N}$, and we have $y^{N}=N\left(a^{j}, 1\right)$ if $j$ is even and $N\left(a^{j}, b\right)$ if $j$ is odd. However $(a, b)$ maps $B$ to $N(a, b)$ and hence $j=1$, and so $k$ is odd (since $j, k$ have the same parity). Also $M \cap K=\left\langle\left(a^{2}, 1\right)\right\rangle$ is transitive on each $M$-orbit, and the $M$-orbits are therefore the cosets $M\left(1, b^{\ell}\right)$ for even $\ell \in \mathbb{Z}_{s}$, and $M\left(a, b^{\ell}\right)$ for odd $\ell \in \mathbb{Z}_{s}$. As in Notation 3.1. $(a, b)$ maps $C=1^{M}=M$ to $C^{+}=y^{M}=M\left(a, b^{k}\right)$. However $(a, b)$ maps $C$ to $M(a, b)$ and hence $k=1$. Thus $S_{0}=\left\{(a, b),\left(a^{-1}, b\right)\right\}$.

It follows that each vertex $\left(a^{k}, b^{\ell}\right)$ has out-neighbours $\left(a^{k \pm 1}, b^{\ell+1}\right)$, and so the map $f:\left(a^{k}, b^{\ell}\right) \mapsto(k, \ell)$ defines a graph isomorphism from $\Gamma$ to the graph $\Gamma^{+}(r, s)$ of Construction 2.6. Also the map $(a, b) \mapsto \mu \nu,\left(a^{2}, 1\right) \mapsto \mu^{2},(c, 1) \mapsto \sigma$ extends to an isomorphism $\varphi^{\prime}$ from $G$ to the group $G^{+}(r, s)$ of Construction 2.6, and $f, \varphi^{\prime}$ define an isomorphism from $\left(\Gamma^{\prime}, G\right)$ to $\left(\Gamma^{+}(r, s), G^{+}(r, s)\right)$.

3.2. Case $\Gamma_{M}$ is $G$-unoriented. We identify $G$ with $G \varphi$, and we first derive a short explicit list of possibilities for $G$.

Lemma 3.5. Using Notation 3.1, identify $G$ with $G \varphi$, and assume that $\Gamma_{M}=C_{s}$ is $G$-unoriented. Then $G_{x}=\langle(c, d)\rangle \cong Z_{2}$, and one of the following holds.

(i) $|V \Gamma|=2 r s, M=\langle(a, 1),(c, 1)\rangle, N=\langle(1, b),(1, d)\rangle$, and $G=M \times N$;

(ii) $r, s$ are both even, $|V \Gamma|=r s, M=\left\langle\left(a^{2}, 1\right),(c, 1)\right\rangle, N=\left\langle\left(1, b^{2}\right),(1, d)\right\rangle$, and $G=\langle M, N,(a, b)\rangle$

(iii) $r, s$ are both even, $|V \Gamma|=\frac{r s}{2}, M=\left\langle\left(a^{2}, 1\right)\right\rangle, N=\left\langle\left(1, b^{2}\right)\right\rangle$, and $G=\langle M, N$, $\left.\left(a, d^{\delta} b\right),(c, d)\right\rangle$, where $\delta=0$ or 1 ;

(iv) $|V \Gamma|=r s, G=(M \times N) \cdot G_{x}$ with $M \times N$ regular on $V \Gamma$, where either $M=\langle(a, 1)\rangle$ or $r$ is even and $M=\left\langle\left(a^{2}, 1\right),(a c, 1)\right\rangle$, and either $N=\langle(1, b)\rangle$ or $s$ is even and $N=\left\langle\left(1, b^{2}\right),(1, b d)\right\rangle$.

Proof. By Lemma 2.1, the subgroup of all elements of $G$ fixing setwise each $N$ orbit, or each $M$-orbit, is equal to $N$, or $M$, respectively, and both $N$ and $M$ are semiregular on $V \Gamma$. Since $G$ is edge-transitive on $\Gamma, G_{x}$ contains an element $h$ that interchanges $y$ and $y^{\prime}$, and hence $h$ interchanges the $N$-orbits $B^{+}$and $B^{-}$. Thus $h^{\Gamma_{N}}=c$ and $G_{x} \cap N=N_{x}$ has index 2 in $G_{x}$. Since $N$ is semiregular it follows that $N_{x}=1$ and so $G_{x}=\langle h\rangle \cong Z_{2}$. Similarly $h$ interchanges $C^{+}$and $C^{-}$so that $h^{\Gamma_{M}}=d$. This implies that $h=(c, d)$, proving the first assertion.

Next we study the setwise stabiliser $N_{C}$ of $C=x^{M}$ in $N$. Since $N \cap M=1$ we have $N_{C} \cong N_{C}^{\Gamma_{M}} \leq G_{C}^{\Gamma_{M}}=\langle d\rangle$, and it follows that $N_{C} \leq\langle(1, d)\rangle$. Similarly 
$M_{B} \leq\langle(c, 1)\rangle$. Note that $(1, d) \in N_{C}$ if and only if $(c, 1) \in M_{B}$ (since $\left.(c, d) \in G_{x}\right)$; in particular $N_{C}=1$ if and only if $M_{B}=1$. Also we may assume, without loss of generality, that $z, z^{\prime}$ lie in $B^{+}, B^{-}$respectively (see Notation 3.1).

Suppose that $(1, d) \in N_{C}$ and let $x^{\prime}:=x^{(1, d)}$. Note that $x^{\prime} \neq x$ (since $N$ is semiregular), and that $x^{\prime}=x^{(c, d)(1, d)}=x^{(c, 1)}$. Thus $x, x^{\prime} \in x^{N} \cap x^{M}=B \cap C$. Since $N^{\Gamma_{M}}$ is a normal subgroup of $G^{\Gamma_{M}}=\langle b, d\rangle$ containing $d$, it follows that $N^{\Gamma_{M}}$ contains $d d^{b}=d b^{-1} d b=b^{2}$, so $N$ is either $\left\langle\left(1, b^{2}\right),(1, d)\right\rangle$ with $s$ even, or $\langle(1, b),(1, d)\rangle$. Thus $|G|=2 r s$ or $4 r s$, and $|V \Gamma|=r s$ or $2 r s$, and hence $|M|=r$ or $2 r$, respectively. Since $(c, 1) \in M$, a similar argument identifies the possibilities for $M$. If $|G|=4 r s$ then (i) holds. If $|G|=2 r s$ then $r, s,|V \Gamma|, M, N$ are as in (ii) and $M \times N$ has index 2 in $G$. Since $a \in G^{\Gamma_{N}}, G$ contains an element of the form $g=\left(a, g^{\prime}\right)$ for some $g^{\prime}$ and, adjusting $g$ by an element of $N$, we may assume that $g^{\prime}=1$ or $b$. Since $M$ does not contain $(a, 1)$ it follows that $g=(a, b)$ and (ii) holds.

Suppose now that $N_{C}=1$, so also $M_{B}=1$. Then $N^{\Gamma_{M}}$ is a semiregular normal subgroup of $G^{\Gamma_{M}}=\langle b, d\rangle=D_{2 r}$, and $N^{\Gamma_{M}}$ does not contain $d$, so we have the following possibilities for $N$ : either $N=\left\langle\left(1, b^{t}\right)\right\rangle$ for some divisor $t$ of $s$, or $s$ is even and $N=\left\langle\left(1, b^{2}\right),(1, b d)\right\rangle$ and in this latter case we set $t=1$. Then $|N|=\frac{s}{t}$, $|G|=\left|G^{\Gamma_{N}}\right| \cdot|N|=\frac{2 r s}{t},|V \Gamma|=\frac{r s}{t}$, and so $|M|=\frac{r}{t}$. Hence $t$ divides $\operatorname{gcd}(r, s)$ and a similar argument shows that either $M=\left\langle\left(a^{t}, 1\right)\right\rangle$, or $r$ is even, $t=1$, and $M=\left\langle\left(a^{2}, 1\right),(a c, 1)\right\rangle$. In all cases $K:=M \times N$ is semiregular on $V \Gamma$ (as it does not contain $(c, d))$ and has $t$ orbits of size $\frac{r s}{t^{2}}$. Now each $K$-orbit is a disjoint union of $\frac{r}{t}$ orbits of $N$, and it follows that an $N$-orbit and an $M$-orbit meet in at most one vertex. In particular $B^{+} \cap C^{+}=\{y\}$. This implies that $z \notin C^{+}$(since we are assuming that $z \in B^{+}$) and hence $z \in C^{-}$and $z^{\prime} \in C^{+}$. Now $y^{K}$ contains $C^{+}=y^{M}$ and hence contains $z^{\prime}$, which implies that $B^{-}=\left(y^{\prime}\right)^{N}=\left(z^{\prime}\right)^{N} \subset\left(z^{\prime}\right)^{K}=y^{K}$. Hence $y^{K}$ contains $\left\{y, y^{\prime}, z, z^{\prime}\right\}$, and it follows, since $K \unlhd G$, that all edges from vertices in $x^{K}$ go to vertices in $y^{K}$, and since $\Gamma$ is connected this implies that $K$ has at most two orbits in $V \Gamma$. Thus $t=1$ or 2 . If $t=1$, then $K$ is regular so $G=K . G_{x}$ as in (iv). Thus we may assume that $t=2$. Then $r, s, M, N,|V \Gamma|$ are as in (iii) and $K$ has index 4 in $G$. Since $G^{\Gamma_{N}}$ contains $a, G$ contains an element of the form $g=\left(a, g^{\prime}\right)$ with $g^{\prime} \in\langle b, d\rangle$. Now $N$ contains $\left(1, b^{2}\right)$ so we may assume that $g^{\prime}=d^{\delta} b^{\delta^{\prime}}$ where each of $\delta, \delta^{\prime}$ is 0 or 1 . The subgroup $M$ does not contain $(a, 1)$, and so the element $g^{\prime} \neq 1$. Also if $g^{\prime}=d$ then $M$ would contain $g(c, d)=(a c, 1)$, which is not the case. Hence $g=(a, b)$ or $(a, d b)$, so $G$ is as in (iii).

Now we analyse cases Lemma 3.5(i)-(iv) separately.

Lemma 3.6. If Lemma 3.5(i) holds then $r$ and $s$ are both odd and $(\Gamma, G) \cong$ $\left(\Gamma_{2}(r, s), G_{2}(r, s)\right)$, as in Construction 2.10.

Proof. Suppose that Lemma 3.5(i) holds. Then $K:=\langle(a, 1), N\rangle$ is a regular normal (index 2) subgroup of $G$, and as in Lemma 3.4 we may assume that $\Gamma=\operatorname{Cay}\left(K, S_{0} \cup\right.$ $S_{0}^{-1}$ ) for a 2-element subset $S_{0} \subseteq K \backslash\{1\}$, with $S_{0} \cap S_{0}^{-1}=\emptyset$ such that $K=\left\langle S_{0}\right\rangle$, $x=1_{K}, S_{0}=\left\{y, y^{\prime}\right\}$. Now $K$ acts by right multiplication, $G_{x}=\langle(c, d)\rangle \leq \operatorname{Aut}(K)$ acts naturally on $V \Gamma$, and $S_{0}$ is a $G_{x^{-}}$-orbit. Thus $y=\left(a^{i}, d^{\delta} b^{j}\right)$ for some $i \in \mathbb{Z}_{r}, j \in$ $\mathbb{Z}_{s}, \delta=0$ or 1 . This means that $y^{\prime}=y^{(c, d)}=\left(a^{-i}, d^{\delta} b^{-j}\right)$ and since $S_{0}=\left\{y, y^{\prime}\right\}$ generates $K$, it follows that $\delta=1, i$ and $j$ are nonzero, and $\operatorname{gcd}(i, r)=1$.

Since $N \leq K$, the $N$-orbits are the cosets $N\left(a^{k}, 1\right)$ for $k \in \mathbb{Z}_{r}$, and as in Notation 3.1. $(a, 1)$ maps $B=1^{N}=N$ to $B^{+}=y^{N}=N\left(a^{i}, 1\right)$. However $(a, 1)$ maps $B$ to $N(a, 1)$ and hence $i=1$. Thus $y=\left(a, d b^{j}\right)$. To determine the orbits of $M$, we 
note that $(c, 1)=(c, d)(1, d) \in M$ maps $u \in K$ to $u^{(c, d)}(1, d)$ and so the $M$-orbit containing $\left(1, b^{k}\right)$ is the set product $\langle(a, 1)\rangle\left\{\left(1, b^{k}\right),\left(1, d b^{k}\right)\right\}$. By Notation 3.1 $(1, b)$ maps $C=1^{M}$ to $C^{+}=y^{M}=\langle(a, 1)\rangle\left\{\left(1, b^{j}\right),\left(1, d b^{j}\right)\right\}$. However $(1, b)$ maps $C$ to $\langle(a, 1)\rangle\{(1, b),(1, d b)\}$ and hence $j=1$. Thus $S_{0}=\left\{(a, d b),\left(a^{-1}, d b^{-1}\right)\right\}$ and since $S_{0}$ generates $K$ we see that both $r$ and $s$ must be odd.

Finally, defining $f: V \Gamma \rightarrow X_{2}$ by $\left(a^{i}, b^{j}\right) \mapsto(i, j)_{0},\left(a^{i}, d b^{j}\right) \mapsto(i, j)_{1}$, and the group isomorphism $\varphi: G \rightarrow G_{2}(r, s)$ extending $(a, 1) \mapsto \mu,(1, b) \mapsto \nu,(c, 1) \mapsto$ $\sigma \tau,(1, d) \mapsto \tau$, we obtain an isomorphism $(f, \varphi)$ from the pair $(\Gamma, G)$ to the pair $\left(\Gamma_{2}(r, s), G_{2}(r, s)\right)$ in Construction 2.10 .

Lemma 3.7. The case Lemma 3.5 (ii) leads to no examples, while if Lemma 3.5(iii) holds, then $\delta=1$ and $(\Gamma, G) \cong\left(\Gamma^{+}(r, s), H^{+}(r, s)\right)$, as in Construction 2.8 .

Proof. Here $r, s$ are both even. The approach is similar to that of the previous lemma. If Lemma 3.5(ii) holds, let $K:=\langle(a, b), N\rangle$, and if Lemma 3.5(iii) holds, let $K:=\left\langle\left(a, d^{\delta} b\right), N\right\rangle$ (where $\delta=0$ or 1 ). In either case $K$ is a normal subgroup of $G$ of index 2 , and $K$ is regular on vertices (since $K \cap G_{x}=1$, implying that $G=K G_{x}$ ). Thus we may assume that $\Gamma=\operatorname{Cay}\left(K, S_{0} \cup S_{0}^{-1}\right)$ for a 2-element generating set $S_{0}$ for $K$ such that $S_{0} \cap S_{0}^{-1}=\emptyset$, and we may identify $x=1_{K}, S_{0}=\left\{y, y^{\prime}\right\}$.

Suppose first that Lemma 3.5(ii) holds. Then $y=\left(a^{i}, b^{j} d^{\delta^{\prime}}\right)$, for some $i \in$ $\mathbb{Z}_{r}, j \in \mathbb{Z}_{s}, \delta^{\prime} \in \mathbb{Z}_{2}$, with $i, j$ of the same parity. Now $S_{0}$ is a $G_{x}$-orbit so $y^{\prime}=$ $y^{(c, d)}=\left(a^{-i}, b^{-j} d^{\delta^{\prime}}\right)$. Since $S_{0} \cap S_{0}^{-1}=\emptyset$, we have $y^{\prime} \neq y^{-1}$, and hence $\delta^{\prime}=1$. However $S_{0}$ is then contained in the proper subgroup $\left\langle\left(a^{2}, 1\right),\left(1, b^{2}\right),\left(a^{i}, b^{j} d\right)\right\rangle$ of index 2 in $K$, contradicting the fact that $S_{0}=\left\{y, y^{\prime}\right\}$ generates $K$.

Thus Lemma3.5(iii) holds. Suppose first that $\delta=0$. Then as $y \in K$, we have $y=$ $\left(a^{i}, b^{j}\right)$ for some $i \in \mathbb{Z}_{r}, j \in \mathbb{Z}_{s}$ of the same parity. This implies, as in the previous paragraph, that $y^{\prime}=y^{(c, d)}=\left(a^{-i}, b^{-j}\right)$ is equal to $y^{-1}$, which is a contradiction. Thus $\delta=1$. If $y=\left(a^{i}, b^{j}\right)$ with both $i, j$ even, then we again find $y^{\prime}=y^{-1}$, a contradiction. Hence $y=\left(a^{i}, d b^{j}\right)$ with both $i, j$ odd. Since $N \leq K$, the $N$-orbits are the cosets $N\left(a^{2 k}, 1\right)$ and $N\left(a^{2 k+1}, d b\right)$, for $0 \leq k<r / 2$, and as in Notation 3.1 the element $(a, d b) \in G$ maps $B=1^{N}=N$ to $B^{+}=y^{N}=N\left(a^{i}, d b^{j}\right)=N\left(a^{i}, d b\right)$ (since $\left.\left(1, b^{2}\right) \in N\right)$. However $(a, d b) \in K$ acts by right multiplication, and maps $B$ to $N(a, d b)$. Hence $i=1$ and $y=\left(a, d b^{j}\right)$. Also $M$ lies in $K$, and the $M$-orbits are the cosets $M\left(1, b^{2 k}\right)$ and $M\left(a, d b^{2 k+1}\right)$, for $0 \leq k<s / 2$. As in Notation 3.1 . the element $(a, d b) \in G$ maps $C=1^{M}=M$ to $C^{+}=y^{M}=M\left(a, d b^{j}\right)$. However $(a, d b) \in K$ acts by right multiplication, and maps $C$ to $M(a, d b)$. Hence $j=1$ and $y=(a, d b), y^{\prime}=y^{(c, d)}=\left(a^{-1}, d b^{-1}\right)$.

Each vertex $w$ of $\Gamma=\operatorname{Cay}\left(K, S_{0} \cup S_{0}^{-1}\right)$ has out-neighbours $y w, y^{\prime} w$, so for $i, j$ even, $w=\left(a^{i}, b^{j}\right)$ has out-neighours $\left(a^{i+1}, d b^{j+1}\right)$ and $\left(a^{i-1}, d b^{j-1}\right)$, while for $i, j$ odd, $w=\left(a^{i}, d b^{j}\right)$ has out-neighours $\left(a^{i+1}, b^{j-1}\right)$ and $\left(a^{i-1}, b^{j+1}\right)$. Thus the map $f$ : $V \Gamma \rightarrow X^{+}$defined by $\left(a^{i}, b^{j}\right) \mapsto(i, j)$ if $i, j$ are both even, and $\left(a^{i}, d b^{j}\right) \mapsto(i, j)$ if $i, j$ are both odd, determines a graph isomorphism from $\Gamma$ to $\Gamma^{+}(r, s)$, which maps each oriented edge $w \rightarrow y w, w \rightarrow y^{\prime} w$ of $\Gamma$ to an oriented edge of $\Gamma^{+}(r, s)$ with the edgeorientation of Construction 2.8. Also the map $\left(a^{2}, 1\right) \mapsto \mu^{2},\left(1, b^{2}\right) \mapsto \nu^{2},(c, d) \mapsto$ $\tau,(a, d b) \mapsto \tau \sigma \mu \nu$, extends to a group isomorphism $\varphi: G \rightarrow H^{+}(r, s)$, and we obtain an isomorphism $(f, \varphi)$ from the pair $(\Gamma, G)$ to the pair $\left(\Gamma^{+}(r, s), H^{+}(r, s)\right)$ in Construction 2.8 .

Lemma 3.8. If Lemma 3.5 (iv) holds then either 
(a) $r$ is odd and $s$ is even, $M=\langle(a, 1)\rangle, N=\left\langle\left(1, b^{2}\right),(1, b d)\right\rangle$, and $(\Gamma, G) \cong$ $(\Gamma(r, s), H(r, s))$ of Construction 2.8; or

(b) $r$ is even and $s$ is odd, $M=\left\langle\left(a^{2}, 1\right),(a c, 1)\right\rangle, N=\langle(1, b)\rangle$, and $(\Gamma, G) \cong$ $(\Gamma(s, r), H(s, r))$ of Construction 2.8.

Proof. Suppose that Lemma 3.5(iv) holds, so $|M|=r,|N|=s,|V \Gamma|=r s$. There are two possibilities for $M$ and two for $N$. For any of these $M, N$, the group $K:=M \times N$ has index 2 in $G$ and acts regularly on the vertices of $\Gamma$. Hence we may assume that $\Gamma=\operatorname{Cay}\left(K, S_{0} \cup S_{0}^{-1}\right)$ for a 2-element generating set $S_{0}$ for $K$ such that $S_{0} \cap S_{0}^{-1}=\emptyset$, and we may identify $x=1_{K}, S_{0}=\left\{y, y^{\prime}\right\}$.

Now $y=m n$ for some $m \in M, n \in N$, and $y^{\prime}$ is the image of $y$ under conjugation by $(c, d) \in G_{x}$. Since $y^{\prime} \neq y^{-1}$, it is not possible for both $m^{(c, d)}=m^{-1}$ and $n^{(c, d)}=n^{-1}$ to hold. Therefore $M, N$ are not both cyclic. Interchanging $r$ and $s$ if necessary, we may assume that $n^{(c, d)} \neq n^{-1}$, and it follows from Lemma 3.5(iv) that $s$ is even and $N=\left\langle\left(1, b^{2}\right),(1, b d)\right\rangle$. We will prove that (a) holds. (In the case where $m^{(c, d)} \neq m^{-1}$ it will then follow, on interchanging $r$ and $s$ in our arguments, that (b) holds.)

Since $n^{(c, d)} \neq n^{-1}$, the element $n$ satisfies $n=\left(1, d b^{j}\right)$ for some $j \in \mathbb{Z}_{s}$, and we have $y=m\left(1, d b^{j}\right), y^{\prime}=m^{(c, d)}\left(1, d b^{-j}\right)$. Now $K$ acts by right multiplication and $M \leq K$, so the $M$-orbits are $C_{2 k}:=M\left(1, b^{2 k}\right)$ and $C_{2 k+1}:=M\left(1, d b^{2 k+1}\right)$ for $0 \leq k<\frac{s}{2}$. The vertex $x$ lies in $C=C_{0}$, and $y$ lies in the image $C^{+}$of $C$ under the action of the element $(c, b)=(1, b d)(c, d) \in G$ (see Notation 3.1, since this element lies in $G$ and induces $b$ on $\left.\Gamma_{M}\right)$. However $(c, b)$ maps $C$ to $(M(1, b d))^{(c, d)}=$ $M\left(1, b^{-1} d\right)=M(1, d b)$, and since this set must contain $y=m\left(1, d b^{j}\right)$, we have $j=1$. Thus $y=m(1, d b)$.

The $N$-orbits are the subsets $N u$ for $u \in M$. The vertex $x$ lies in $B=N$, and $y$ lies in the image $B^{+}$of $B$ under the action of the element $(a, 1)$ if $M=\langle(a, 1)\rangle$, or $(a, d)=(a c, 1)(c, d)$ if $M=\left\langle\left(a^{2}, 1\right),(a c, 1)\right\rangle$ (see Notation 3.1. since these elements lie in $G$ and induce $a$ on $\left.\Gamma_{N}\right)$. In the second case, the element $(a, d)$ maps $B$ to $(N(a c, 1))^{(c, d)}=N\left(a^{-1} c, 1\right)=N(c a, 1)$, and since this set must contain $y=$ $m(1, d b)$ we have $m=(c a, 1)$. However this implies that $y=(c a, d b)$ which has order 2 and hence $y^{-1}=y \in S_{0}$, a contradiction. Therefore $M=\langle(a, 1)\rangle$. The element $(a, 1)$ maps $B$ to $B^{+}=N(a, 1)$ and since this set contains $y$ it follows that $m=(a, 1)$ and $y=(a, d b), y^{\prime}=\left(a^{-1}, d b^{-1}\right)$. Now the fact that $\left\langle S_{0}\right\rangle=K$ implies that $r$ must be odd.

It remains to identify the graph-group pair. The oriented edges of $\Gamma$ are the pairs $u \rightarrow y u$ and $u \rightarrow y^{\prime} u$ for $u \in K$. An easy computation shows that, for each $i \in \mathbb{Z}_{r}$ and for $0 \leq j<\frac{s}{2}$,

$$
\begin{aligned}
\left(a^{i}, b^{2 j}\right) & \rightarrow\left(a^{i+1}, d b^{2 j+1}\right) & \text { and } & \left(a^{i}, b^{2 j}\right) & \rightarrow & \left(a^{i-1}, d b^{2 j-1}\right), \\
\left(a^{i}, d b^{2 j+1}\right) & \rightarrow\left(a^{i+1}, b^{2 j}\right) & \text { and } & \left(a^{i}, d b^{2 j+1}\right) & \rightarrow & \left(a^{i-1}, b^{2 j+2}\right) .
\end{aligned}
$$

It follows that the bijection $f: K \rightarrow \mathbb{Z}_{r} \times \mathbb{Z}_{s}$ given by $f:\left(a^{i}, b^{2 j}\right) \mapsto(i, 2 j)$ and $f:\left(a^{i}, d b^{2 j+1}\right) \mapsto(i, 2 j+1)$ defines a graph isomorphism from $\Gamma$ to the graph $\Gamma(r, s)$ such that the oriented edges $u \rightarrow y u$ and $u \rightarrow y^{\prime} u$ of $\Gamma$ are mapped to oriented edges according to the edge-orientation defined in Construction 2.8. Also the map $\varphi$ given by

$$
\varphi:(a, 1) \mapsto \mu, \varphi:\left(1, b^{2}\right) \mapsto \nu^{2}, \varphi:(1, d b) \mapsto \tau \sigma \nu, \varphi:(c, d) \mapsto \tau
$$


extends to an isomorphism from $G$ to the group $H(r, s)$ of Construction 2.8. Moreover the pair $(f, \varphi)$ determines an isomorphism from $(\Gamma, G)$ to $(\Gamma(r, s), H(r, s))$.

3.3. Proof of Theorem 2, Let $(\Gamma, G) \in \mathcal{O G}(4)$ have independent cyclic normal quotients $\Gamma_{N}, \Gamma_{M}$ of orders $r, s$ respectively. We may assume that $N, M$ consists of all elements of $G$ which fix setwise each $N$-orbit, or each $M$-orbit, respectively. Part (a) of Theorem 2 follows from Lemma 3.1(a), and we may assume that $\Gamma_{N}$ is $G$-unoriented. Part (b) of Theorem 2 follows from Lemma 3.1(b), and (as explained just before Notation (3.1) for the rest of the proof we may assume that $N \cap M=1$. If $\Gamma_{M}$ is $G$-oriented then, by Lemmas 3.2, 3.3, and 3.4, $\left|G_{x}\right|=2$ and line 1 or 2 of Table 1 holds. If $\Gamma_{M}$ is $G$-unoriented then, by Lemmas 3.5, 3.6, 3.7 and 3.8 $\left|G_{x}\right|=2$ and one of the lines $3,4,5$ or 6 of Table 1 holds. This completes the proof.

\section{WeAK METACIRCULANTS WITH INDEPENDENT CYCLIC NORMAL QUOTIENTS}

First we see that under Hypothesis 1.1, it is only the graphs in Class I of [10] for which the quotient $\Gamma_{R}$, defined as in Section 1, can possibly be a cyclic normal quotient.

Lemma 4.1. Suppose that Hypothesis 1.1 holds.

(a) If $\Gamma_{R}$ is a normal quotient of $(\Gamma, G)$, then $\Gamma$ lies in Class I or IV of [10] relative to $(\rho, \lambda)$, and if $\Gamma_{R}$ is cyclic then $\Gamma$ lies in Class $I$.

(b) Conversely, for $\Gamma$ in Class I or IV relative to $(\rho, \lambda)$, either $H$ is regular on vertices, or $\Gamma$ lies in Class I and $(\Gamma, H) \in \mathcal{O} \mathcal{G}(4)$ with $\Gamma_{R}$ an $H$-oriented cyclic normal quotient.

Proof. (a) Suppose that $\Gamma_{R}$ is equal to $\Gamma_{N}$ for some $N \unlhd G$ (that is to say the sets of $R$-orbits and $N$-orbits on vertices are the same). By [1, Thm 1.1], there are no edges joining vertices in the same $R$-orbit, and moreover there is a constant $k$ such that, if there is an edge between some vertex in the $R$-orbit $X_{i}$ and some vertex in $X_{j}$, then each vertex of $X_{i}$ is adjacent to exactly $k$ vertices in $X_{j}$. These conditions are not satisfied for Classes II and III of [10, Section 3], and hence only Classes I and IV can correspond to normal quotients of $(\Gamma, G)$. Moreover, since the quotients for Class IV have valency 4 , it is only the quotients for Class I which can arise as cyclic normal quotients.

(b) Suppose that $\Gamma$ is in Class I or IV of 10 relative to $(\rho, \lambda)$, and that $H$ is not regular on vertices. Then $H_{x} \neq 1$ for $x$ in the $R$-orbit $X_{1}$, and since the action induced by $H$ on the $R$-orbits is cyclic (induced by $\lambda$ ), $H_{x}$ fixes each $R$-orbit setwise. If $H_{x}$ fixed each vertex adjacent to $x$ then, since $\Gamma$ is connected, it would follow that $H_{x}$ would fix all vertices, contradicting our assumption that $H_{x} \neq 1$. Thus $H_{x}$ moves some vertex $y$ adjacent to $x$, say $y \in X_{2}$, and hence $X_{2}$ contains at least two vertices adjacent to $x$. For $\Gamma$ in Class IV, the four vertices adjacent to $x$ lie in four distinct $R$-orbits and hence we conclude that $\Gamma$ lies in Class I of [10], and that two $R$-orbits distinct from $X_{1}$, namely $X_{2}$ and, say, $X_{3}$, each contain two vertices adjacent to $x$. Thus $H_{x}$ interchanges the two vertices in $\Gamma(x) \cap X_{2}$, and since $H$ preserves the edge-orientation, it follows that $H$ is edge-transitive, so $(\Gamma, H) \in \mathcal{O G}(4)$, with $\Gamma_{R}$ a cyclic normal quotient. Finally the fact that $H_{x}$ interchanges the two vertices in $\Gamma(x) \cap X_{2}$ implies that $\Gamma_{R}$ is an $H$-oriented cycle. (This last fact can also be deduced from the proofs of [10, Lemmas 4.2 and 4.3] although it is not explicitly stated there.) 
We give examples to show that, for a given $(\Gamma, G) \in \mathcal{O G}(4)$, different pairs $(\rho, \lambda)$ can lead to different behaviours for the quotient $\Gamma_{R}$, even with the same subgroup $H=\langle\rho, \lambda\rangle$.

Construction 4.2. Let $r$ be an odd integer, $r \geq 3$, and let $\Gamma=\Gamma(r, r)$ and $G=$ $G(r, r)$, as in line 1 of Table 3. Then, using Lemma 2.7 we see that $(\Gamma, G) \in \mathcal{O G}(4)$, and that, for $\rho, \lambda$ as in (a), (b) or (c) below, $\Gamma$ is an $(r, r)$-metacirculant relative to $(\rho, \lambda), H:=\langle\rho, \lambda\rangle=\langle\mu, \nu\rangle$ is regular, and the quotient $\Gamma_{R}$, for $R:=\langle\rho\rangle$, is a cycle $C_{r}$.

(a) Let $\rho=\mu \nu$ and $\lambda=\mu$. Then $\Gamma_{R}$ is $G$-oriented but is not a normal quotient of $(\Gamma, G)$ since $N_{G}(R)=H$. (The $R$-orbits are $\left\{(i+\ell, \ell) \mid \ell \in \mathbb{Z}_{r}\right\}$, for $i \in \mathbb{Z}_{r}$.)

(b) Let $\rho=\nu$ and $\lambda=\mu$. Then $\Gamma_{R}$ is a normal quotient of $(\Gamma, G)$ that is not $G$-oriented.

(c) Let $\rho=\mu$ and $\lambda=\nu$. Then $\Gamma_{R}$ is a $G$-oriented normal quotient of $(\Gamma, G)$.

Now we study the case where $(\Gamma, G)$ has independent cyclic normal quotients and where $\Gamma_{R}$ is a $G$-oriented normal quotient, so $\Gamma$ is of Class I of [10, by Lemma 4.1]

Lemma 4.3. Suppose that Hypothesis 1.1 holds with $\Gamma_{R} \cong C_{m}$ a G-oriented normal quotient of $(\Gamma, G)$, and that $(\Gamma, G)$ has independent cyclic normal quotients. Then for a possibly different pair $(\rho, \lambda)$ of elements from $H$, the same conditions all hold and $(\Gamma, G)$ has a possibly different pair of independent cyclic normal quotients, one of which is $\Gamma_{R}$.

Proof. Let $\Gamma_{M}, \Gamma_{N}$ be independent cyclic normal quotients of $(\Gamma, G)$, for normal subgroups $M, N$ of $G$. We may assume that $M$ (respectively $N$ ) is equal to the kernel of the $G$-action on $\Gamma_{M}\left(\right.$ respectively $\left.\Gamma_{N}\right)$. Let $\bar{\Gamma}=\Gamma_{M \cap N}, \bar{G}=G /(M \cap N)$, etc., as in Lemma 3.1. Since $\Gamma_{M}, \Gamma_{N}$ are independent, $(\bar{\Gamma}, \bar{G}) \in \mathcal{O G}(4)$. Moreover, by Theorem 2. the stabiliser $G_{x}$ of a vertex $x$ has order 2. Thus $G=H G_{x}$ and $|G: H| \leq 2$, since $H$ is vertex-transitive.

Since $\Gamma_{R}$ is a normal quotient of $(\Gamma, G)$, there is a normal subgroup $\hat{R}$ of $G$, containing $R$, with the same vertex-orbits as $R$, and such that $\hat{R}$ is equal to the kernel of the $G$-action on $\Gamma_{R}$. Moreover, since $\Gamma_{R}=\Gamma_{\hat{R}}$ is $G$-oriented, the group $G / \hat{R}$ induced on $\Gamma_{R}$ is cyclic of order $m$, and is therefore equal to the cyclic group induced by $\langle\lambda\rangle$ on $\Gamma_{R}$, so $G=\langle\hat{R}, \lambda\rangle$.

Suppose first that some $R$-orbit is a union of $j$ of the $M$-orbits in $V \Gamma$, where $j \geq 1$. Then each $R$-orbit is a union of $j$ of the $M$-orbits, and $\Gamma_{R}$ is isomorphic to a quotient of $\Gamma_{M}$. Since $m \geq 3$ and $\Gamma_{R}$ is $G$-oriented, it follows that $\Gamma_{M}$ is also $G$-oriented. Thus $G$ induces a regular cyclic group on $\Gamma_{M}$, and as $H$ is vertextransitive, $H$ induces the same group, so $G=\left\langle M, \lambda^{\prime}\right\rangle$ for some element $\lambda^{\prime} \in H$. Moreover we may choose $\lambda^{\prime}$ such that $\lambda, \lambda^{\prime}$ induce the same action on $\Gamma_{R}$, and hence $\lambda^{\prime}=\lambda \rho^{i}$ for some $i$. Also $M \cap H$ is the subgroup $\left\langle\rho^{\prime}\right\rangle$, where $\rho^{\prime}=\rho^{j}$, and Hypothesis 1.1 holds with $\left(\rho^{\prime}, \lambda^{\prime}\right)$ in place of $(\rho, \lambda)$, and $M \cap H$ in place of $R$ (noting that $\rho^{\lambda}=\rho^{r}$ implies that $\left(\rho^{\prime}\right)^{\lambda^{\prime}}=\left(\rho^{\prime}\right)^{r}$, and $\operatorname{gcd}(r, n)=1$ implies that $\left.\operatorname{gcd}\left(r, o\left(\rho^{\prime}\right)\right)=1\right)$. Since $\Gamma_{M}$ is $G$-oriented, $M$ contains $G_{x}$ by Lemma2.1(b). Hence $M=(M \cap H) G_{x}, \Gamma_{M \cap H}=\Gamma_{M}$ (the 'new $\Gamma_{R}$ '), and all the assertions hold relative to $\left(\rho^{\prime}, \lambda^{\prime}\right)$, and the normal quotients $\Gamma_{N}, \Gamma_{M \cap H}$.

Thus we may assume that $\hat{R}$ does not contain $M$. Suppose next that $M$ properly contains $\hat{R}$, that is, $\Gamma_{M}$ is a quotient of $\Gamma_{R}$. Then $\Gamma_{N}, \Gamma_{R}$ are independent cyclic 
normal quotients and all the assertions hold without changing $(\rho, \lambda)$. So now we may assume also that $M$ does not contain $\hat{R}$. Let $T:=R \cap M$ and note that $T \leq H$ since $R \leq H$. If $\Gamma_{T}$ is not cyclic then $\Gamma_{M}, \Gamma_{R}$ are independent cyclic normal quotients and all the assertions hold without changing $(\rho, \lambda)$. Assume then that $\Gamma_{T}$ is cyclic. Since $\Gamma_{M}$ is a quotient of $\Gamma_{T}$, the pair $\Gamma_{N}, \Gamma_{T}$ are independent cyclic normal quotients, and as $T \subseteq R$, each $R$-orbit is a union of $T$-orbits. Thus the arguments of the previous paragraph may be used to replace $(\rho, \lambda)$ by a new pair $\left(\rho^{\prime}, \lambda^{\prime}\right)$ from $H$, and replace $R$ by $T$, and $\Gamma_{R}$ by $\Gamma_{T}$, so that all assertions hold for the independent cyclic normal quotients $\Gamma_{N}, \Gamma_{T}$.

Finally we prove Corollary 1.2

Proof of Corollary 1.2. Suppose that Hypothesis 1.1 holds with $\Gamma_{R} \cong C_{m}$ a $G$ oriented cyclic normal quotient of $(\Gamma, G) \in \mathcal{O G}(4)$, where $m \geq 3$. Suppose also that, as in Theorem 2. $(\Gamma, G)$ has independent cyclic normal quotients $\Gamma_{N} \cong C_{r}, \Gamma_{M} \cong$ $C_{s}$, where $N, M$ are the kernels of the $G$-actions on $\Gamma_{N}, \Gamma_{M}$ respectively. By Theorem 2, we may assume that $\Gamma_{N}$ is $G$-unoriented, and by Lemma 4.3, we may assume that one of the independent cyclic normal quotients is $\Gamma_{R}$. (Note that, from the proof of Lemma 4.3, $\Gamma_{R}$ may be replaced by a cyclic $G$-oriented normal quotient of order a proper multiple of the original $m$ in Hypothesis 1.1, but in the exposition we can, and will, continue to use $m$ as the order of $\Gamma_{R}$.) Since $\Gamma_{R}$ is $G$-oriented, we have $\Gamma_{R}=\Gamma_{M} \cong C_{m}$, so $m=s$.

By Theorem 2 again, setting $T:=M \cap N, \bar{G}:=G / T$ and $\bar{\Gamma}:=\Gamma_{T}$, the pair $(\Gamma, G)$ is a normal cover of $(\bar{\Gamma}, \bar{G}) \in \mathcal{O G}(4)$, and $\bar{\Gamma}, \bar{G}$ and $\bar{\Gamma}_{\bar{M}} \cong \Gamma_{M} \cong C_{\bar{m}}$ are as in line 1 or 2 of Table 1 where $\bar{M}=M / T$ and $\bar{m}=m=s$. Let the order of $\bar{\Gamma}$ be $\bar{m} \bar{n}$. Then $\bar{n}$ is $r$ or $r / 2$ for line 1 or 2 of Table 1, respectively. Thus, so far we have proved that $\bar{m}, \bar{n}, \bar{\Gamma}, \bar{G}$, and the conditions on $r, s$ are as in the appropriate line of Table 2. Finally we prove that $\bar{\Gamma}$ is a weak $(\bar{m}, \bar{n})$-metacirculant.

Consider first line 1 of Table 2 Then $\bar{G}=\langle\mu, \nu, \sigma\rangle$ where $\mu, \nu, \sigma$, are as in Definition 2.1. It is easy to check that $\bar{\Gamma}$ is a weak $(s, r)$-metacirculant relative to $(\mu, \nu)$, so the assertions for line 1 of Table 2 all hold. The graph $\bar{\Gamma}$ is the graph $X_{o}(s, r ; 1)$ defined in [10, Example 2.1] (although in [10] both of $r, s$ are assumed to be odd). If indeed both of $r, s$ are odd then $\bar{\Gamma}$, and hence also $\Gamma$, is $\frac{1}{2}$-arc transitive (see [10, Theorem 4.1] and its proof).

Now consider line 2 of Table2. Then $\bar{G}=\left\langle\mu^{2}, \mu \nu, \sigma\right\rangle$ where again $\mu, \nu, \sigma$, are as in Definition 2.1. This time $\bar{\Gamma}$ is a weak $(s, r / 2)$-metacirculant relative to $\left(\mu^{2}, \mu \nu\right)$, so the assertions for line 2 of Table 2 all hold. The graph $\bar{\Gamma}$ is the graph $X_{e}(s, r / 2 ; 1,0)$ defined in [10, Example 2.2] (although in [10, $r / 2$ is assumed to be even and at least 4). Thus Corollary 1.2 is proved.

\section{REFERENCES}

[1] J. A. Al-Bar, A. Al-Kenani, N. M. Muthana, and C. E. Praeger, Finite edge-transitive oriented graphs of valency four: a global approach. Submitted. 2014. arXiv: 1405.5427

[2] B. Alspach and T.D. Parsons, A construction for vertex-transitive graphs, Canad. J. Math. 34 (1982) 307-318.

[3] B. Alspach, D. Marušič, and L. Nowitz, Constructing graphs which are $\frac{1}{2}$-transitive, J. Aust. Math. Soc. A 56 (1994), 1-12.

[4] I. Antončič and P. Šparl, Classification of quartic half-arc-transitive weak metacirculants of girth at most 4 Disc. Math. 339 (2016), 931-945.

[5] C. Godsil and G. F. Royle, Algebraic Graph Theory, Springer-Verlag, New York, 2001. 
[6] D. Marušič, Recent developments in half-transitive graphs, Discrete Math 182 (1998), 219231.

[7] D. Marušič, Half-transitive group actions on finite graphs of valency 4, J. Combin. Theory (B) 73 (1998), 41-76.

[8] D. Marušič and R. Nedela, Maps and half-transitive graphs of valency 4, Eur. J. Combin. 19 (1998), 345-354.

[9] D. Marušič and C. E. Praeger. Tetravalent graphs admitting half-transitive group actions: alternating cycles, J. Combin. Theory (B) $\mathbf{7 5}$ (1999), 188-205.

[10] D. Marušič and P. Šparl, On quartic half-arc-transitive metacirculants, J. Algebr. Comb. 28 (2008), 365-395.

[11] D. Marušič and A. O. Waller, Half-transitive graphs of valency 4 with prescribed attachment numbers, J. Graph Theory 34 (2000), 89-99.

[12] M. Šajna, Half-transitivity of some metacirculants, Discrete Math. 185 (1998) 117-136.

[13] P. Šparl, A classification of tightly attached half-arc-transitive graphs of valency 4. J. Comb. Theory, Ser. B 98 (2008), 1076-1108.

[14] P. Šparl, On the classification of quartic half-arc-transitive metacirculants. Disc. Math. 309 (2009), 2271-2283.

[15] P. Šparl, Quartic half-arc-transitive weak metacirculants of Class III (in preparation).

[16] P. Šparl, Almost all quartic half-arc-transitive weak metacirculants of Class II are of Class IV. Disc. Math. 310 (2010), 1737-1742.

(All authors) King Abdulaziz University, Jeddah, Saudi Arabia

(Cheryl E. Praeger) Also affiliated with: Centre for the Mathematics of Symmetry and Computation, School of Mathematics and Statistics M019, The University of Western Australia, 35 Stirling Highway, Crawley, WA 6009, Australia

E-mail address, Jehan A. Al-bar: jalbar@kau.edu.sa; jaal [underscore] bar@hotmail.com

E-mail address, Ahmad N. Al-kenani: analkenani@kau.edu.sa; aalkenani10@hotmail.com

E-mail address, Najat M. Muthana: nmuthana@kau.edu.sa;

E-mail address, Najat M. Muthana (second email): najat [underscore]muthana@hotmail.com

E-mail address, Cheryl E. Praeger: cheryl.praeger@uwa.edu.au 